\title{
First scientific observations with MEGARA at GTC
}

\section{Gil de Paz, A., Carrasco, E., Gallego, J., Iglesias-Páramo, J., Cedazo, R., et al.}

A. Gil de Paz, E. Carrasco, J. Gallego, J. Iglesias-Páramo, R. Cedazo, M. L. García-Vargas, X. Arrillaga, J. L. Avilés, A. Bouquin, J. Carbajo, N. Cardiel, M. A. Carrera, A. Castillo-Morales, E. Castillo-Domínguez, S. Esteban San Román, D. Ferrusca, P. Gómez-Álvarez, R. IzazagaPérez, B. Lefort, J. A. López-Orozco, M. Maldonado, I. Martínez-Delgado, I. Morales-Durán, E. Mujica, G. Páez, S. Pascual, A. Pérez-Calpena, P. Picazo, A. Sánchez-Penim, E. SánchezBlanco, S. Tulloch, M. Velázquez, J. M. Vílchez, J. Zamorano, A. L. Aguerri, D. Barrado y Navascues, S. R. Berlanas, E. Bertone, A. Cava, C. Catalán-Torrecilla, J. Cenarro, M. Chávez, B. T. Dullo, M. García, J. García-Rojas, J. Guichard, R. González-Delgado, R. Guzmán, A. Herrero, N. Huélamo, D. H. Hughes, J. Jiménez-Vicente, C. Kehrig, R. A. Marino, I. Márquez, J. Masegosa, D. Mayya, J. Méndez-Abreu, M. Mollá, C. Muñoz-Tuñón, M. Peimbert, P. G. Pérez-González, E. Pérez-Montero, M. Rodríguez, J. M. Rodríguez-Espinosa, L. Rodríguez Merino, L. Rodríguez-Muñoz, D. Rosa-González, J. Sánchez-Almeida, C. Sánchez-Contreras, P. Sánchez-Blázquez, S. F. Sánchez, A. Sarajedini, S. Silich, S. SimónDíaz, G. Tenorio-Tagle, E. Terlevich, R. Terlevich, S. Torres-Peimbert, I. Trujillo, Y. Tsamis, O. Vega, "First scientific observations with MEGARA at GTC," Proc. SPIE 10702, Ground-based and Airborne Instrumentation for Astronomy VII, 1070217 (6 July 2018); doi: 10.1117/12.2313299

Event: SPIE Astronomical Telescopes + Instrumentation, 2018, Austin, Texas, United States 


\title{
First scientific observations with MEGARA at GTC
}

A. Gil de Paz*a , E. Carrasco ${ }^{\text {b }}$, J. Gallego ${ }^{\text {a }}$ J. Iglesias-Páramo ${ }^{\mathrm{c}}$, R. Cedazo ${ }^{\mathrm{d}}$, M.L. García Vargas ${ }^{\mathrm{e}}$, X.

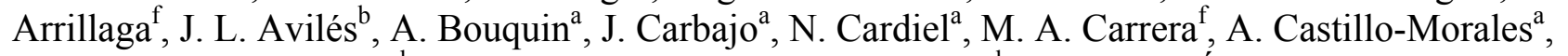

E. Castillo-Domínguez ${ }^{b}$, S. Esteban San Román ${ }^{a}$, D. Ferrusca ${ }^{b}$, P. Gómez-Álvarez e R. Izazaga-

Pérez $^{\mathrm{b}}$, B. Lefort ${ }^{\mathrm{e}}$, J.A. López-Orozco ${ }^{\mathrm{a}}$, M. Maldonado ${ }^{\mathrm{e}}$, I. Martínez-Delgado ${ }^{\mathrm{e}}$, I. Morales Durán ${ }^{\mathrm{c}}$, E.

Mujica $^{\mathrm{e}}$, G. Páez ${ }^{\mathrm{g}}$, S. Pascual ${ }^{\mathrm{a}}$, A. Pérez-Calpena ${ }^{\mathrm{e}}$, P. Picazo ${ }^{\mathrm{a}}$, A. Sánchez-Penim ${ }^{\mathrm{a}}$, E. Sánchez-

Blanco $^{\mathrm{e}}$, S. Tulloch ${ }^{\mathrm{e}}$, M. Velázquez ${ }^{\mathrm{b}}, \mathrm{J}_{\text {.M. Vílchez }}^{\mathrm{c}}$, J. Zamorano ${ }^{\mathrm{a}}$, A.L. Aguerri ${ }^{\text {h,i }}$, D. Barrado y

Naváscues $^{\mathrm{j}}$, S. R. Berlanas ${ }^{\mathrm{h}, \mathrm{i}}$, E. Bertone ${ }^{\mathrm{b}}$, A. Cavak ${ }^{\mathrm{k}}$, C. Catalán-Torrecilla ${ }^{\mathrm{a}}$, J. Cenarro ${ }^{1}$, M. Chávez $^{\mathrm{b}}$, B. T. Dullo ${ }^{\mathrm{a}}$, M. García ${ }^{\mathrm{j}}$, J. García-Rojas ${ }^{\mathrm{h}, \mathrm{i}}$, J. Guichard ${ }^{\mathrm{b}}$, R. González-Delgado ${ }^{\mathrm{c}}$, R. Gúzman ${ }^{\mathrm{m}}$, A.

Herrero $^{\text {h,i }}$, N. Huélamo ${ }^{\mathrm{j}}$, D. H. Hughes ${ }^{\mathrm{b}}$, J. Jiménez-Vicente ${ }^{\mathrm{n}}$, C. Kehrig ${ }^{\mathrm{c}}$, R.A. Marino ${ }^{\mathrm{o}}$, I. Márquez ${ }^{\mathrm{c}}$,

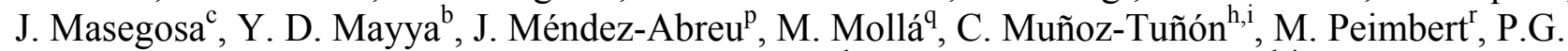

Pérez-González ${ }^{a}$, E. Pérez Montero ${ }^{c}$, M. Rodríguez ${ }^{\mathrm{b}}, \mathrm{J}_{\text {.M. Rodríguez-Espinosa }}{ }^{\mathrm{h}, \mathrm{i}}$, L. Rodríguez-

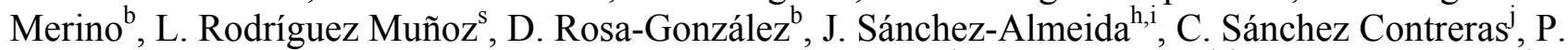
Sánchez-Blázquez, S. F. Sánchez ${ }^{\mathrm{r}}$, A. Sarajedini ${ }^{\mathrm{m}}$, S. Silich ${ }^{\mathrm{b}}$, S. Simón-Díaz,i $^{\mathrm{h}, \mathrm{G}}$ G. Tenorio-Tagle ${ }^{\mathrm{b}}$, E. Terlevich ${ }^{\mathrm{b}}, \mathrm{R}$. Terlevich ${ }^{\mathrm{b}}, \mathrm{S}$. Torres-Peimbert ${ }^{\mathrm{r}}$, I. Trujillo ${ }^{\mathrm{h}, \mathrm{i}}$, Y. Tsamis ${ }^{\mathrm{u}}$, O. Vega $^{\mathrm{b}}$

${ }^{\mathrm{a}}$ Universidad Complutense de Madrid (UCM, Spain), ${ }^{\mathrm{b}}$ Instituto Nacional de Astrofísica, Óptica y

Electrónica (INAOE, Mexico), ${ }^{\mathrm{C} I n s t i t u t o}$ de Astrofísica de Andalucía (IAA-CSIC, Spain),

${ }^{\mathrm{d}}$ Universidad Politécnica de Madrid (UPM, Spain), ${ }^{\mathrm{e}}$ FRACTAL SLNE (Spain), ${ }^{\mathrm{f}}$ AVS SL, ${ }^{\mathrm{g}}$ Centro de Investigaciones en Óptica (Mexico), ${ }^{\mathrm{h}}$ Instituto de Astrofísica de Canarias (Spain), ${ }^{\mathrm{i}}$ Universidad de

La Laguna (Spain), ${ }^{\mathrm{j}}$ Centro de Astrobiología (Spain), ${ }^{\mathrm{k}}$ University of Geneva $(\mathrm{CH}),{ }^{1}$ Centro de Estudios de Física del Cosmos de Aragón (Spain), ${ }^{\mathrm{m}}$ University of Florida (USA), ${ }^{\mathrm{n}}$ Universidad de Granada (Spain), ${ }^{\circ}$ Institut für Astronomie, ETH Zurich (Switzerland), ${ }^{\mathrm{p}}$ University of St. Andrews, ${ }^{\mathrm{q}}$ CIEMAT (Spain), ${ }^{\mathrm{r}}$ Universidad Autónoma de México (Mexico), ${ }^{\mathrm{s}}$ Osservatorio Astronomico di Padova (Italy), ${ }^{\mathrm{t} U n i v e r s i d a d ~ A u t o ́ n o m a ~ d e ~ M a d r i d ~(S p a i n), ~ " D e p a r t m e n t ~ o f ~ P h y s i c s ~ a n d ~ A s t r o n o m y, ~}$ University College London (UK)

\begin{abstract}
On June 25th 2017, the new intermediate-resolution optical IFU and MOS of the 10.4-m GTC had its first light. As part of the tests carried out to verify the performance of the instrument in its two modes (IFU \& MOS) and 18 spectral setups (identical number of VPHs with resolutions $\mathrm{R}=6000-20000$ from 0.36 to 1 micron) a number of astronomical objects were observed. These observations show that MEGARA@GTC is called to fill a niche of high-throughput, intermediateresolution IFU \& MOS observations of extremely-faint narrow-lined objects. Lyman- $\alpha$ absorbers, star-forming dwarfs or even weak absorptions in stellar spectra in our Galaxy or in the Local Group can now be explored to a new level. Thus, the versatility of MEGARA in terms of observing modes and spectral resolution and coverage will allow GTC to go beyond current observational limits in either depth or precision for all these objects. The results to be presented in this talk clearly demonstrate the potential of MEGARA in this regard.
\end{abstract}

Keywords: Intermediate-resolution IFU \& MOS, Optical spectrograph, Resolved stellar populations, Cosmic web, Lyman- $\alpha$ forest, Kinematics and dynamics, Nearby galaxies, Optical fibers

*agil@ucm.es; phone +34 91394 5179; fax +34 91394 4635; http://guaix.fis.ucm.es/megara/

Ground-based and Airborne Instrumentation for Astronomy VII, edited by Christopher J. Evans, Luc Simard, Hideki Takami, Proc. of SPIE Vol. 10702, 1070217 - (c) 2018 SPIE · CCC code: 0277-786X/18/\$18 · doi: 10.1117/12.2313299 


\section{INTRODUCTION}

MEGARA is the new facility instrument of the $10.4 \mathrm{~m}$ GTC telescope in the Observatory of Roque de los Muchachos (La Palma, Spain). It offers GTC with intermediate-to-high spectral resolutions in the visible (365-970 nm) ranging between $\mathrm{R}=6,000$ and $\mathrm{R}=20,000$ in both Integral Field Spectroscopy (IFS) and Multi-Object Spectroscopy (MOS) modes. The Integral Field Unit (IFU), which is called Large Compact Bundle (LCB hereafter), covers a field of $12.5 \times 11.3 \operatorname{arcsec}^{2}$ using 567 hexagonal spaxels of 0.62 arcsec in size plus 56 sky spaxels of equal size distributed in 8 bundles of 7 fibers distributed in the outskirts of the field at about 2 arcmin from the center of the LCB. The MOS makes use of a set of 92 robotic positioners each hosting a minibundle of 7 spaxels also of $0.62 \operatorname{arcsec}$ in size each spaxel. These can patrol overlapping circular regions of $28 \mathrm{arcsec}$ in diameter. These robotic positioners a distributed in a square region of $3.5 \mathrm{x}$ $3.5 \mathrm{arcmin}^{2}$, which roughly corresponds to the flat and non-vignetted focal plane of GTC at its Folded-Cassegrain F (FCF) focus (see Figure 1). The MOS can reconfigure starting from a list of target potions in matter of roughly a minute to a few minutes, depending on the level of number of overlapping patrol areas to be explored in a given configuration.

The spaxels constituting both the LCB and MOS modes are defined by microlenses that change the focal ratio from $\mathrm{F} / 17$ yielded by the FC-F focus of GTC to F/3 to minimize Focal-Ratio Degradation (FRD) and loss of light at our F/3 collimator. The image of the pupil is then re-imaged by these microlenses on $100 \mu \mathrm{m}$-core fibers.
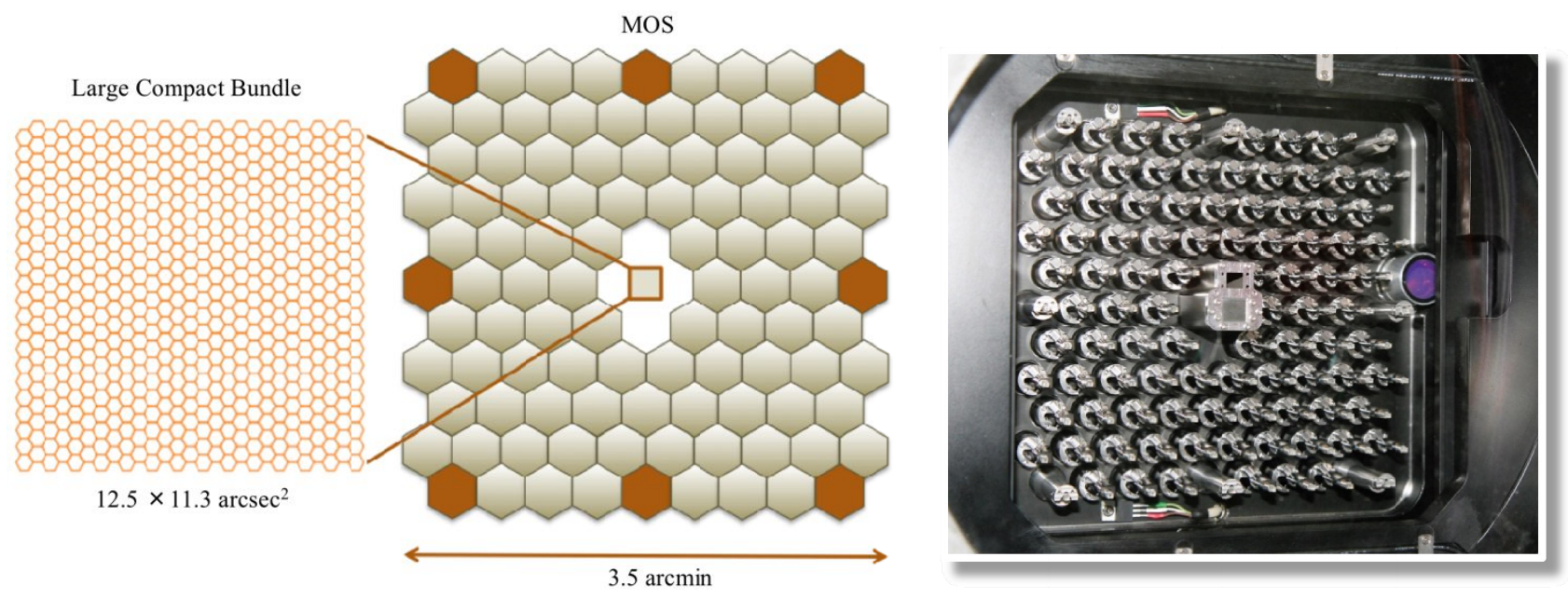

Figure 1: LCB and MOS in the focal plane of MEGARA at the Folded-Cass F focus of GTC. Left: Layout of the monolithic microlens array of the LCB placed at the optical axis of the instrument. Center: Hexagons representing the patrol areas of the 92 robotic positioners of the MEGARA MOS (in light grey) along with the positions of the eight sky bundles that are mounted along the LCB pseudo-slit (in orange). Note that the actual patrol areas are overlapping circular regions of 28 arcsec in diameter, while the distance between adjacent positioners is 24.5 arcsec. Right: MEGARA focal plane before the field lens was installed at the Laboratorio de Instrumentación Cientifica Avanzada (LICA-UCM) laboratory.

Both the LCB and the MOS along with other subsystems (focal-plane cover, Folded-Cassegrain rotator adapter, etc.; see Carrasco et al. $2018^{1}$ for more details) are located at the FC-F focus of GTC. The $623(567+56)$ fibers of the LCB and the 644 fibers of the 92 robotic positioners of the MOS are then routed through the FC-F rotator, on the telescope ring, through the elevation rotator, under the Nasmyth A platform, following a $44.5 \mathrm{~m}$-long path until they reach the MEGARA spectrograph. The MEGARA spectrograph is a fixed-angle $\left(68^{\circ}\right)$ collimator-camera system that is fed by two interchangeable curved pseudo-slits (LCB/MOS). The collimator is an all-refractive $\mathrm{F} / 3$ system composed by 5 lenses ( 1 aspheric singlet and 2 doublets) while the also all-refractive camera is composed by 7 lenses (two doublets, one with a $\mathrm{CaF}_{2}$ lens, and 3 singlets). In between collimator and camera, the spectrograph pupil can host different types of Volume-Phase Holographic (VPH) disperser elements, namely the low- (LR), mid(MR), and high-resolution (HR) VPHs. Six LR VPHs cover the entire optical window at $R=6,000$, while 10 MR VPHs provide also full optical coverage but at $\mathrm{R}=12,000$. Finally, the two HR VPHs allow observing in the 
$\mathrm{H} \alpha+[\mathrm{NII}]$ region and in the $\mathrm{CaT}$ region with $\mathrm{R}=20,000$, although the optical design could in principle accommodate HR VPHs at any other optical wavelength. While the LR VPHs host holographic gelatins sandwiched between flat fused-silica window, MR and HR VPHs also require of the use of large prisms at each side of these flat windows to change the incidence angle on the gelatin and yield higher resolving powers. In Figure 2 we show the resolving power and spectral coverage for each VPH as measured during the integration and commissioning of the instrument.
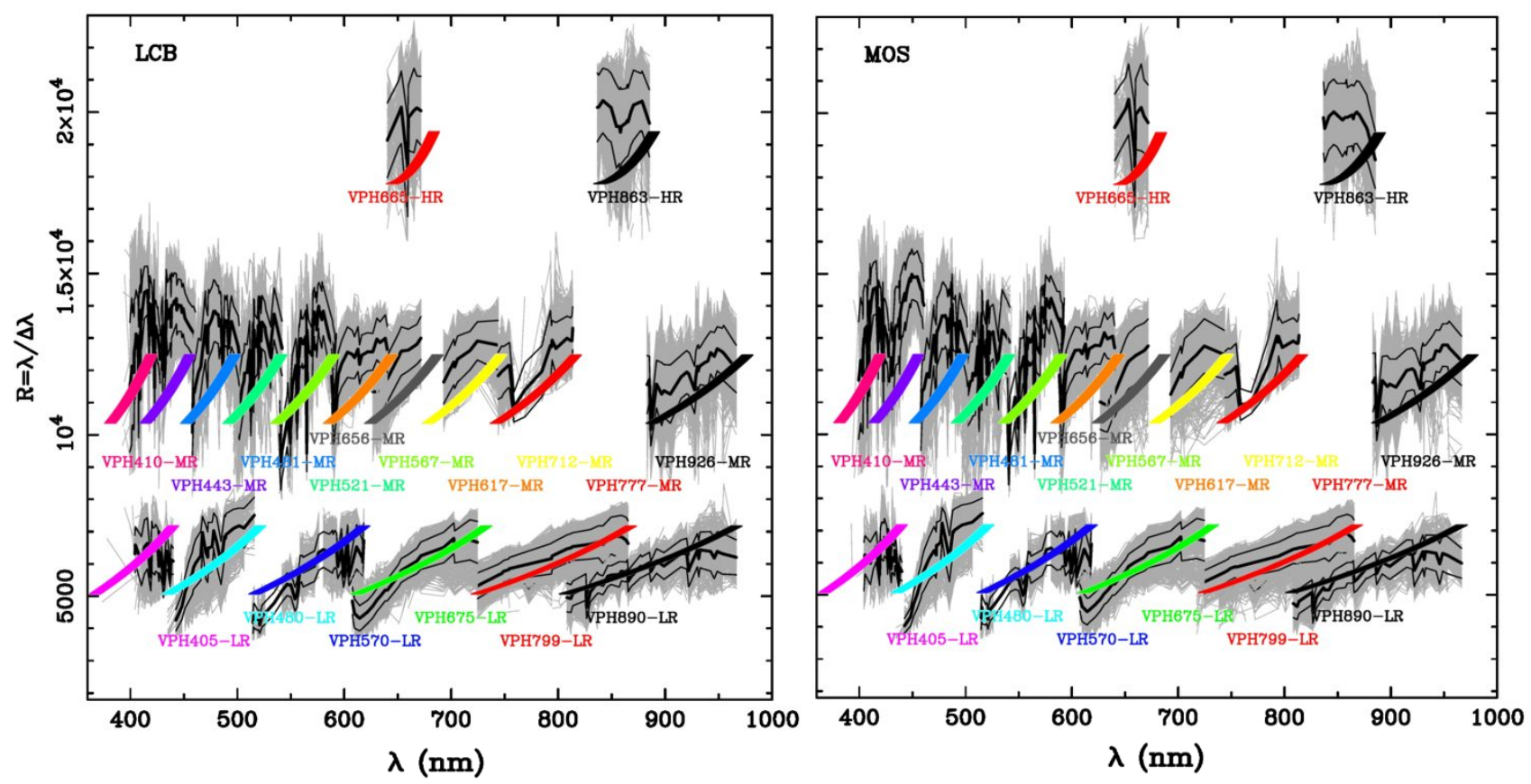

Figure 2: Plots showing the relation between resolving power $\left(\mathrm{R}_{\mathrm{FWHM}}\right)$ and wavelength coverage for all 18 MEGARA VPHs and for the LCB (left) and MOS (right) modes. Design values (colored lines) and measurements (grey lines that correspond to individual fiber spectra, while black thick and thin lines represent the mean and mean $\pm 1 \sigma$ curves when all fiber spectra are used) are both shown.

The deployment of the MEGARA instrument at GTC was accompanied by the installation of a fully functioning Data Reduction Pipeline (DRP hereafter) developed in Python that worked both online at the telescope and offline. The online version of the DRP allowed for on-the-fly data processing, which included bias correction, trimming, fiber tracing and fixed-aperture extraction, fiber-flat and twilight-flat correction and wavelength calibration. The offline processing additionally includes a detailed cross-talk-corrected extraction and absolute flux calibration whenever possible. The

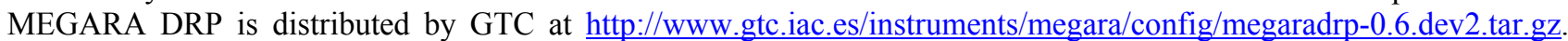
Updates to the DRP can be obtained through github at https://github.com/guaix-ucm/megaradrp.

The final products of the MEGARA DRP are "reduced" Row-Stacked Spectra (RSS hereafter) 2D images including for 623 (644) fiber spectra for the LCB (MOS) mode, all using a common flux calibration and wavelength solution with constant reciprocal dispersion for all fibers. Based on the averaged spectrum of all fibers to be used for sky subtraction (by default all 56 sky fibers in the LCB and all unassigned minibundles in the case of the MOS) the DRP also generates a sky-subtracted "final" RSS spectrum. No combo products combining different spectral setups are yet generated.

The results presented in this contribution were obtained using the online and offline versions of the MEGARA DRP.

In addition, as part of the preparation of the commissioning observations with MEGARA we also made use of a series of tools that constitute the MEGARA Observing Preparation Software Suite (MOPSS) and that included the MEGARA Exposure Time Calculator (ETC, http://gtc-phase2.gtc.iac.es/mect/etc/) and the Fiber-MOS Assignment Tool (FMAT, http://www.gtc.iac.es/instruments/megara/config/megara-fmat.zip) (see two top panels of Figure 3). Also included in MOPSS is the MEGARA quick-look software that runs embedded within the Java GTC inspector along with the MEGARA Control Panel itself (see two bottom panels of Figure 3). 

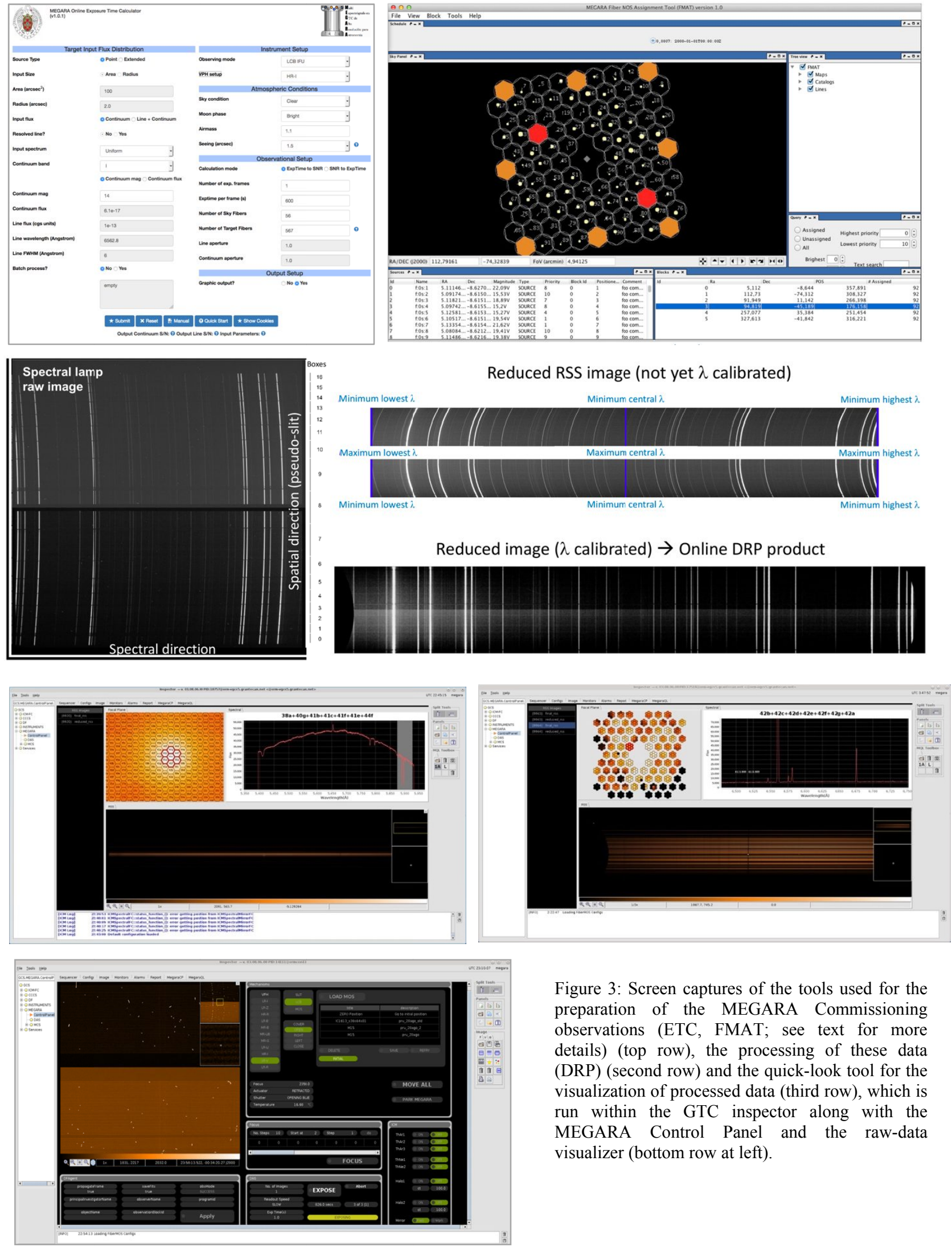

Figure 3: Screen captures of the tools used for the preparation of the MEGARA Commissioning observations (ETC, FMAT; see text for more details) (top row), the processing of these data (DRP) (second row) and the quick-look tool for the visualization of processed data (third row), which is run within the GTC inspector along with the MEGARA Control Panel and the raw-data visualizer (bottom row at left). 
Table 1. MEGARA Main Characteristics.

\begin{tabular}{|l|l|}
\hline IFU (LCB) Field of View & $12.5 \times 11.3 \operatorname{arcsec}^{2}$ \\
\hline MOS Field of View & $92 \times 1.6$ arcsec diameter mini-IFUs in 3.5 x 3.5 $\operatorname{arcmin}^{2}$ \\
\hline Spaxel size for both LCB \& MOS & 0.62 arcsec (diameter of the circle inscribing the hexagon) \\
\hline Wavelength range & $365-970 \mathrm{~nm}$ \\
\hline Spectral resolution ( $\left.\mathrm{R}_{\mathrm{FWHM}}\right)$ & 6,000 (LR VPHs), 12,000 (MRs), 20,000 (HRs; measured) \\
\hline Multiplexing LCB & 567 IFU fibers + 56 (7 fiber x 8 bundles) sky fibers \\
\hline Multiplexing MOS & 644 fibers (7 fiber x 92 bundles) \\
\hline GTC foci & Folded-Cass F (Fiber-MOS) + Nasmyth A (spectrograph) \\
\hline
\end{tabular}

In Table 1 we give a summary of main characteristics of MEGARA. For more details on the instrument the reader is referred to the contribution by Carrasco et al. (2018) ${ }^{1}$ in this meeting and elsewhere ${ }^{2,3,4,5,6}$. From now on we focus on the tasks and scientific observations carried out as part of the commissioning of the MEGARA instrument at GTC.

\section{MEGARA SCIENCE}

As a facility instrument for GTC, MEGARA was designed with the objective of being able to satisfy the needs of a broad range of scientific objectives within the GTC astronomical community, namely Spain, Mexico and University of Florida (USA). This led to the formation of a large and diverse (in terms of areas of research) Science Team mainly constituted by astronomers from GTC partner institutions. Thus, our MEGARA Science Team includes people working on Galactic Science, both stellar astronomy (including exoplanets) and interstellar (ISM) and circumstellar (CSM) media, and on Extragalactic Science, from the study of resolved stellar populations to more distant unresolved galaxies to very first galaxies and the emission from the high-redshift inter-galactic medium (IGM).

One notorious aspect of all these scientific aspects is that their needs are not fulfilled using an instrument based simply on either a single monolithic IFU or a single-fiber/slitlet MOS, both modes are clearly a must. This is now becoming evident when looking to the most recent initiatives in astronomical instrumentation for optical and near-infrared spectroscopy, which are clearly dominated by either multi-IFU (sometimes with multiple combinations of multiplexing and individual FoV) or single, but large-FoV, IFU instruments. Examples of the former are KMOS, WEAVE, MOSAIC while MUSE or KCWI are examples of the latter (see contributions on these instruments as part of these proceedings). As shown in Section 1, MEGARA shares characteristics with some of these instruments as it was designed to meet the requirements of scientists working both on (1) Galactic and extragalactic extended nebulae and (2) compact sources (at least marginally resolved from the ground) clustered in the sky with intermediate-to-high surface densities. Category (1) includes the study of Planetary Nebulae, nearby galaxies, and the high-redshift IGM while in category (2) we can include Galactic open stellar clusters, resolved stellar populations in Local Group galaxies, intermediate-redshift dwarf and starburst galaxies, and high-redshift cluster galaxies. Figure 4 below provides a graphical summary of some of the major scientific objectives of our Science Team regarding the exploitation of MEGARA, going from the study of pre-Planetary Nebulae and anti-center Open Clusters in our own Milky Way to the study of proto-galaxy clusters and rest-frame resonant UV lines from the IGM at very high redshift.

Since these scientific objectives are described in detail elsewhere ${ }^{2,3,4,5,6}$, including a detailed description of the immediate plans for the exploitation of the Guaranteed Time obtained with MEGARA at $\mathrm{GTC}^{1}$, we focus in this contribution on the first observations of astronomical targets carried out during the commissioning of MEGARA at GTC between June and August 2017. The commissioning observations described in Section 3 below were designed to effectively evaluate the instrument specifications and design requirements while providing, at the same time, scientific results that would readily demonstrate the capabilities of MEGARA to the GTC community boosting its demand as part of the Open-Time calls. 


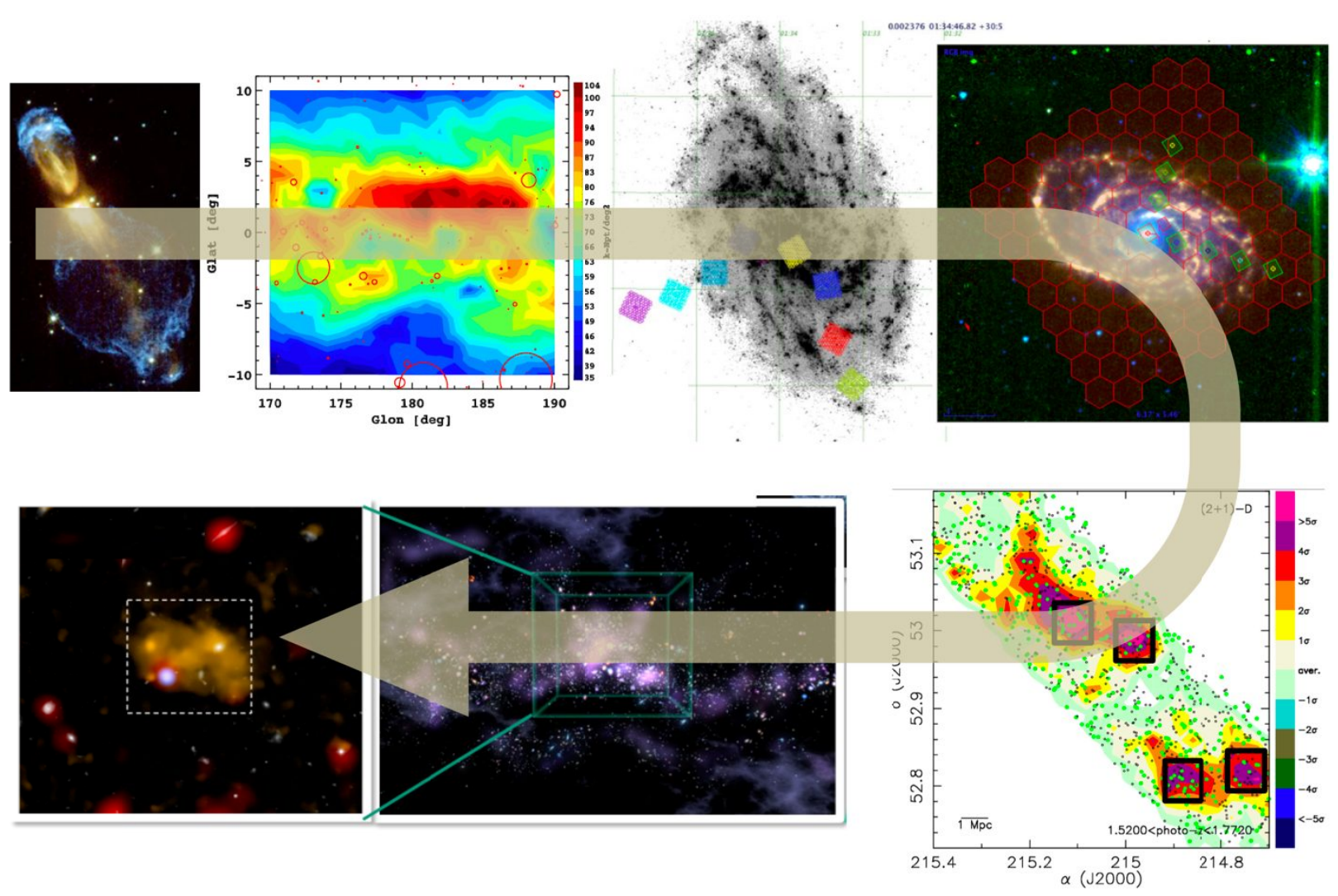

Figure 4: Graphical summary of our broad scientific topics within the MEGARA Science Team, from the study of our own Milky Way to the high-redshift Universe. Following the arrow, we show specific targets that will be the objective of the MEGARA observations to be carried out by our team as a representation of each of these topics. Top row (left to right): prePlanetary Nebula $\mathrm{OH} 231.8+4.2^{7}$, Galactic anti-center Open Clusters, Local Group galaxy M33, nearby galaxy from the $\mathrm{S}^{4} \mathrm{G}$ survey $^{8}$ (the layout of the MOS FoV is shown on top of these two latter objects). Bottom row (right to left): High-redshift proto-galaxy cluster, Lyman- $\alpha$ blob and the Cosmic Web.

\section{COMMISSIONING RESULTS}

Right after the laboratory acceptance on February 2017, the MEGARA team and GRANTECAN (public company in charge of the maintenance and operations of GTC) agreed on both detailed integration and commissioning plans. The latter included both daytime and night-time activities (see Sections 3.1 and 3.2 below). The instrument was packed during the first two weeks of March 2017, leaving the UCM facilities on Saturday, March $18^{\text {th }} 2017$. The instrument left the Iberian Peninsula from the Cádiz port on March $21^{\text {st }}$ and arrived to the La Palma port at El Tinglado on March $24^{\text {th }}$ 2017. Finally, the instrument arrived to GTC on Tuesday, March $28^{\text {th }}$ 2017. After several weeks of intense daytime work (from March $28^{\text {th }}$ to April $7^{\text {th }} 2017$ and from April $17^{\text {th }}$ to April $28^{\text {th }}$ 2017) the instrument was successfully integrated at GTC. A full description of the packing, transport and integration activities of the MEGARA instrument is given in PérezCalpena et al. (2018a) ${ }^{9}$. In this section we present the results of the instrument commissioning exclusively.

\subsection{Daytime commissioning}

The daytime commissioning of MEGARA started on May $8^{\text {th }} 2017$ and lasted for 2 weeks, from May $8^{\text {th }}$ to May $12^{\text {th }}$ and from June $5^{\text {th }}$ to June $9^{\text {th }}$ 2017. A total of 42 daytime commissioning tests were carried out during these periods by a total of 12 people from the MEGARA team and additional support from GTC staff. These tests were split in four large sets, Control System Interface, MEGARA FC-F setup, MEGARA Spectrograph setup, and Instrument configuration $(M o d e+V P H)$ characterization tests. Here we will focus on the last two sets of tests. 


\section{MEGARA Spectrograph setup tests:}

Among this first set of tests we performed the following individual daytime tests. In the vast majority of the cases the instrument was illuminated with arc lamps, of either ThAr or ThNe, depending on the VPH used, and the instrument mode used was the LCB. This is partly due to the fact that GTC did not provide an Instrument Calibration Module (ICM) covering the entire MEGARA FoV until the second daytime commissioning run.

- $\quad$ Pseudo-slit mechanism repeatability: In this test, we verified the stability of the pseudo-slit when commanded to a set of movements and displacements from the collimator entrance and checking that it is able to return to its starting position, so the position of the final spectra is not modified. The procedure included: (1) selecting a VPH (the LR-I VPH in this case), (2) take a reference LCB image with that specific VPH and with its optimal arc lamp, (3) move the pseudo-slit mechanism from LCB to MOS, (4) move the pseudo-slit mechanism back to LCB, (4) take another image and (5) repeat steps (2) through (4) three times. The analysis was carried out over the raw images after applying cosmic ray correction, bias and background subtraction. Several emission lines were selected from the reference image over 6 different spatial and spectral regions obtaining their centroids after fitting a Gaussian profile to each line. From the analysis of the centroids in all images we obtained a maximum difference of $\sim 0.04$ pixels in the centroids, i.e. less than $1 \mu \mathrm{m}$, well below the requirement of $1 / 10$ of the element resolution (equivalent to $6 \mu \mathrm{m}$ on the detector).

- Focus mechanism repeatability: We tested the stability of the focus mechanism by first selecting a grating (the LR-I VPH in this case) and placing the mechanism at best focus position $\mathrm{z}_{0}$ (in $\mu \mathrm{m}$ ) of the selected pupil element (taking into account the operational temperature at that moment). The mechanism is moved several times from $z_{0}$ by $\pm z \mu m$ a moved back to $z_{0}$ and images are taken every time the focus mechanism is moved back to $\mathrm{z}_{0}$. the analysis was carried out over the RAW images after applying cosmic ray correction, bias and background subtraction. As in the previous test, several emission lines were selected from different spatial and spectral regions and centroids were computed (and the difference with those in the reference image analyzed) after fitting a Gaussian profile to each line. The requirement establishes that the centroids of one spectral line and two fibers must be better than a 1/10 of the resolution element, again $6 \mu \mathrm{m}$ on the detector. The analysis of data collected yielded a mean difference between the centroids of $\sim 0.08$ pixels, i.e. less than $1.2 \mu \mathrm{m}$.

- Grating mechanism repeatability: As part of this test we first place one specific pupil element (the LR-R VPH in this case) in the pupil with its optimal focus setup and illuminate the instrument (LCB). At that moment we took a reference image. Then the VPH is placed back in the VPH wheel and the wheel is moved from position 1 to position 10 so this mechanism is moved a total of $\sim 327$ degrees. Later on, the wheel returns to position 1 and the actuator places the VPH at the pupil position again. This was repeated four times and, as in the previous tests, the difference between the centroids is computed at the end of the observing sequence using raw images on which only a cosmic-ray correction, bias and background subtraction reduction steps have been applied. From these results, we derived a mean difference between of centroids of $\sim 0.07$ pixels, i.e. less than $1.1 \mu \mathrm{m}$ and, again, less than the $6 \mu \mathrm{m}$ required.

- Pseudo-slit vignetting: We performed displacements of the pseudo-slit mechanism parallel to the collimator cover spanning a distance from 41540 to $51540 \mu \mathrm{m}$ (from the mechanism reference) for the LCB and from 81540 to $91540 \mu \mathrm{m}$ for the MOS, both in $1000 \mu \mathrm{m}$ steps. The objective here was to map the flux variation in every step and looking for the maximum of the light coming through the spectrograph. That will determine the position where the LCB and MOS pseudo-slits were not vignetted by the collimator aperture. We used the LR-I VPH and flux measurements were done using images illuminated with the ICM halogen lamps. In Figure 5 we show the flux curves as a function of pseudo-slit mechanism position for the LCB (left) and MOS (Right). The resulting nominal positions used from there on were $46540 \mu \mathrm{m}$ for the LCB and $86540 \mu \mathrm{m}$ for the MOS.

- Shutter vignetting: The objective of this test is to determine the optimal position for the instrument rotatory shutter (see Carrasco et al. 2018 ${ }^{1}$ ) in the case of the order-sorter (RED) open position using the LR-I VPH and the ThAr lamp. The other two positions (BLUE and CLOSED) were fixed by the hexagonal geometry of the shutter. In order to determine the least-vignetted rotation position of the shutter, we took images by changing the open position in steps of 25 motor steps, from 1214 to 1439 steps. As for the previous test we measured the flux in six different regions (see Figure 5) both spatially and spectrally. The results are shown in Figure 6, where a maximum (corresponding to the optimal open position for the order-sorter filter) is seen at $\sim 1340$ steps. 


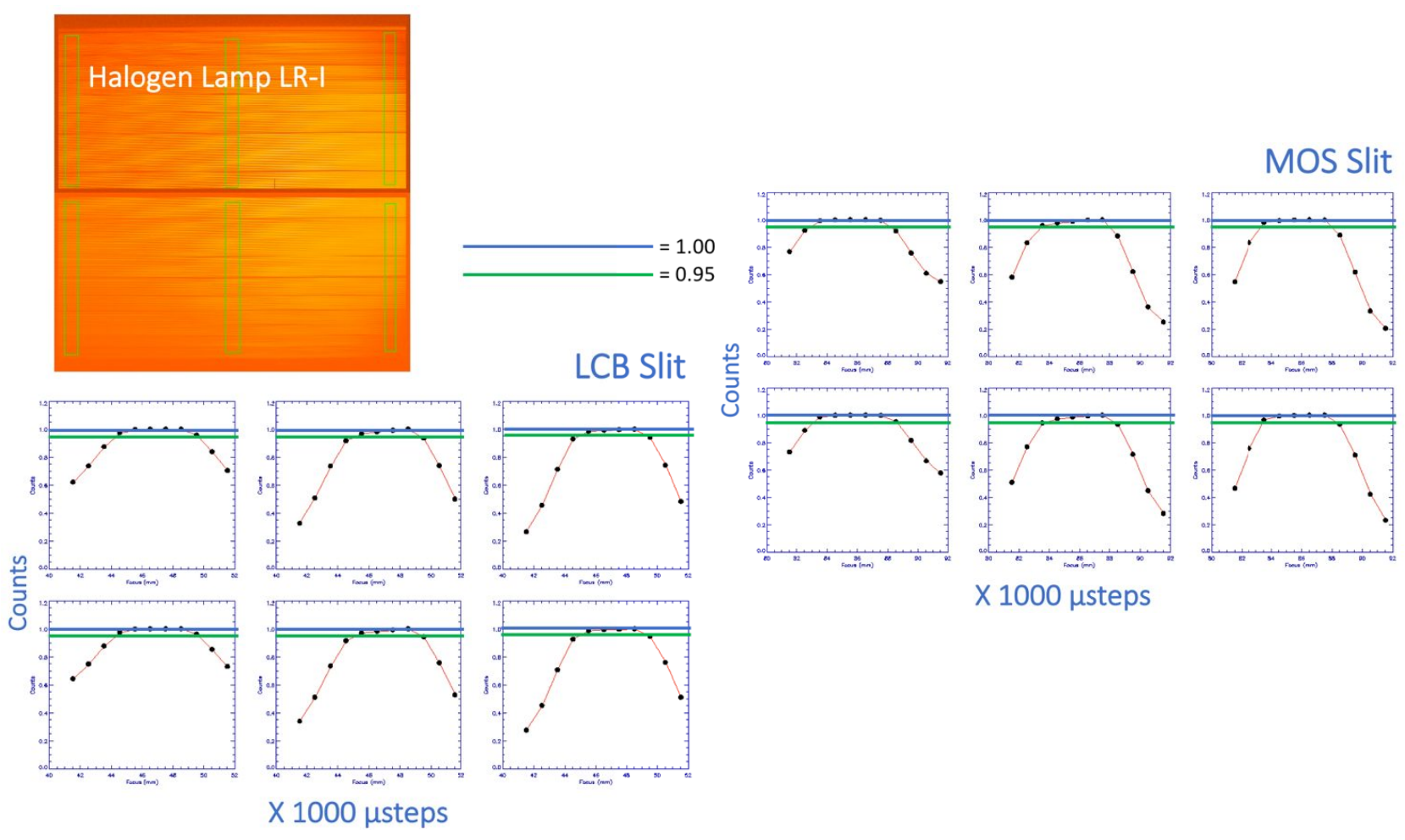

Figure 5: Results of the pseudo-slit vignetting tests for the LCB (bottom left) and MOS (top right). The regions used for evaluating the level of vignetting in different spatial and spectral regions of the MEGARA CCD are also shown (top left).
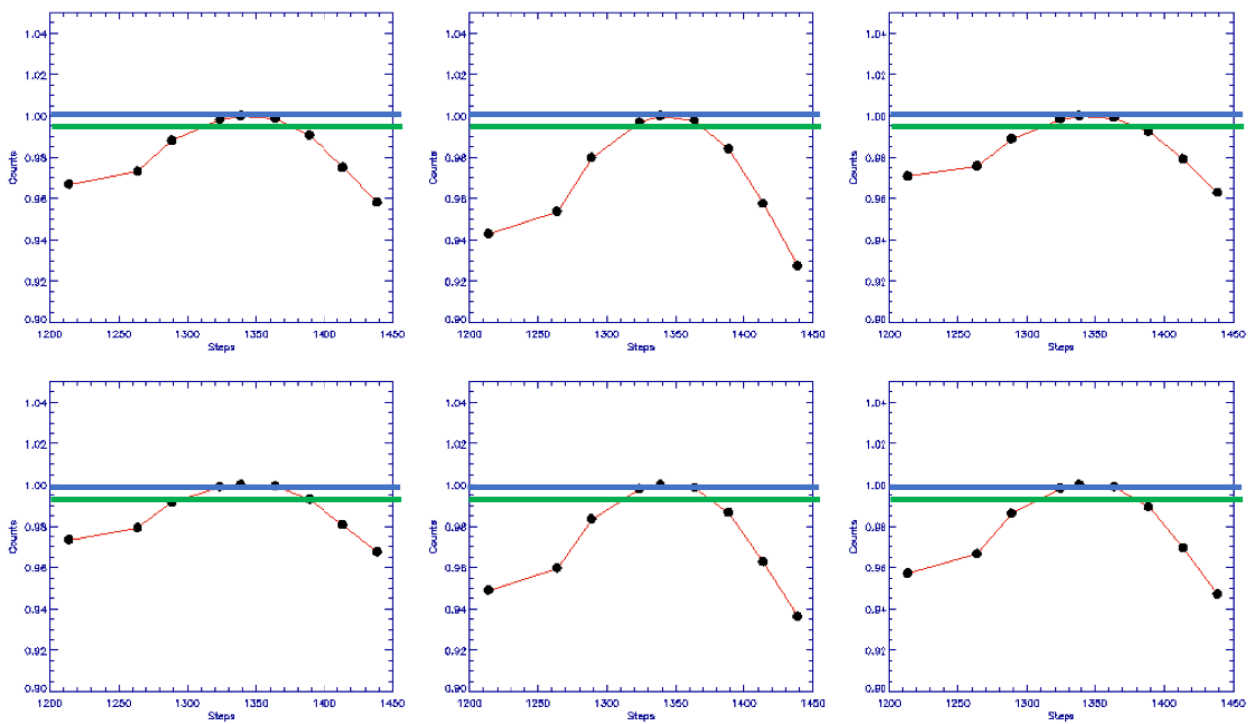

Figure 6: Results of the shutter vignetting test for the order-sorter (RED) open position. The regions used for evaluating the level of vignetting in different spatial and spectral regions of the MEGARA CCD are shown in Figure 5. 
- Pseudo-slit homogeneity: In this test we studied the fiber-to-fiber sensitivity by illuminating the instrument with the system of halogen lamps provided by the ICM. We made used of 5 different VPHs in the MOS observing mode and with different quantities of lamps regulating the intensity as required by each pupil element. For performing the test, we obtained a master fiber flat for each grating calibrated in wavelength and derived profiles collapsing boxes of different sizes of the image in the spectral. What defines the quality and homogeneity in these images is the distribution of light from peak to peak for each fiber. We finally proceeded to find all the local maxima in the profiles and obtaining the difference of illumination between the peaks with higher illumination and those ones that showed a lower light sensitivity. The deviation found from fibers showing the highest sensitivity from those ones with lowest one is $<8 \%$. Specific numbers by VPH are $<8 \%$ in LR-I, $<7 \%$ in LR-R, $<8 \%$ in LR-Z, $<7 \%$ in MR-B and $<7 \%$ in MR-G.

\section{Instrument configuration (Mode+VPH) characterization:}

The goal of this test was to characterize each instrument configuration considering all combinations of LCB and MOS modes and all the 18 MEGARA VPH gratings. Although all these instrument configurations had been previously characterized at LICA-UCM they were reviewed again at the GTC as part of the daytime commissioning tests.

The procedure carried out for each VPH and mode included (1) obtaining a focus sequence to determine the corresponding optimal focus, (2) acquiring bias and (3) halogen- and (4) arc-lamp images at that focus, and (5) to measure the positions and line widths (FWHM) of the spectral lines for deriving (i) the absolute wavelength calibration and (ii) the image quality (in pixels and Ångstroms and, therefore, the $\mathrm{R}_{\mathrm{FWHM}}$ ) both in all fiber spectra (623 in the LCB and 644 in the MOS). Only unresolved lines must be used since otherwise optical errors could be attributed to real spectral widths.

From these measurements we derived the central wavelength, spectral coverage, image quality $\left(\mathrm{EER}_{80}\right)$ and spectral resolution (i.e. $\Delta \lambda=F W H M$ and $\mathrm{R}_{\mathrm{FWHM}}=\lambda / \Delta \lambda$ as a function of wavelength; see Figure 2). We also obtained the optimum focus position (averaged and as a function of position in the detector) and the corresponding best-average-focus arc and flat-field images, extracted arc-lamp spectrum, and trace map. See Table 2 for a compilation of some of these numbers.
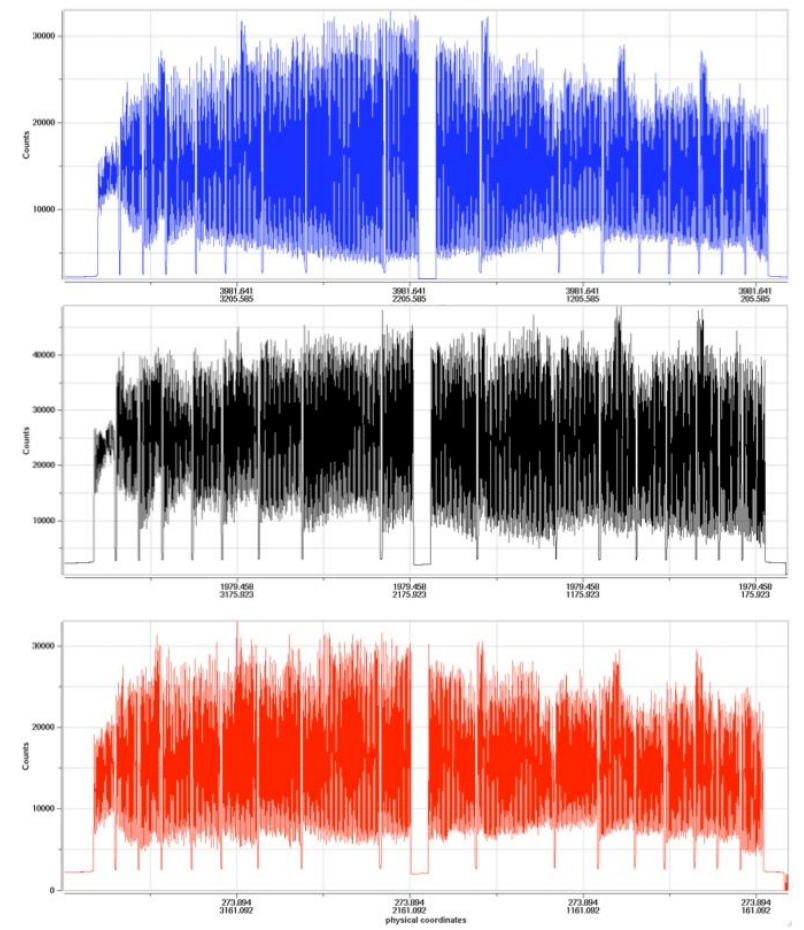

Figure 7: Spatial profile of the raw image of a halogen lamp observation with the LCB in the MR-V VPH. The top, central and bottom panels correspond to 10-pixel wide profiles taken at the blue-end, the central, and red-end of the raw 2D image, respectively. The flux in the inter-fiber rows is dominated by adjacent-fiber cross-talk and not by low-frequency scattering. 
We also estimated the level of scattering in the spatial/spectral direction. In order to evaluate this scattering level, we obtained the spatial profile of a halogen lamp image taken with the MR-V VPH in the LCB mode (see Figure 7). The three panels shown in this figure correspond to the profiles taken at the blue end of the spectrum, the approximate central wavelength, and the red end of the spectrum. The level of the bias corresponds to the count rate measured at the edges of the plots. The flatness of the lower envelope of this flux distribution (which corresponds to the inter-fiber positions) indicates that this flux level is dominated by cross-talk between adjacent fibers and not by low-frequency scattered light. The contamination from scattered light generated by the spectrograph commonly shows a pattern that decreases significantly from the center to the edges of the pseudo-slit or, at the very minimum, shows a clear low-frequency structure. We also note that the count rate in between fibers is about $10-15 \%$ of the peak count rate of each fiber, with 5$7 \%$ coming from each of the two adjacent fibers.

Table 2. Results of the instrument commissioning regarding the spectral performance of the different MEGARA VPHs. $\lambda \min _{1}\left(\lambda \max _{2}\right)$ represents the minimum (maximum) wavelength covered for at least one of the LCB fibers, while $\lambda \mathrm{c}$ represents the central wavelength and $\lambda \min (\lambda \max )$ the minimum (maximum) wavelength common to all LCB fibers.

\begin{tabular}{|c|c|c|c|c|c|c|}
\hline VPH & $\lambda \min _{1}(\AA)$ & $\lambda \min (\AA)$ & $\lambda \mathrm{c}(\AA)$ & $\lambda \max (\AA)$ & $\lambda \max _{2}(\AA)$ & Disp. $(\AA /$ pix $)$ \\
\hline LR-U & 3640,04 & 3654,32 & 4025,90 & 4391,88 & 4417,33 & 0,195 \\
\hline LR-B & 4278,43 & 4332,05 & 4785,32 & 5199,96 & 5232,02 & 0,230 \\
\hline LR-V & 5101,13 & 5143,74 & 5678,63 & 6168,19 & 6206,04 & 0,271 \\
\hline LR-R & 6047,62 & 6096,54 & 6729,61 & 7303,21 & 7379,88 & 0,321 \\
\hline LR-I & 7166,47 & 7224,11 & 7976,31 & 8640,37 & 8822,29 & 0,380 \\
\hline LR-Z & 7978,45 & 8042,74 & 8873,16 & 9634,92 & 9692,58 & 0,421 \\
\hline MR-U & 3911,99 & 3919,81 & 4102,87 & 4282,17 & 4289,11 & 0,092 \\
\hline MR-UB & 4217,44 & 4226,38 & 4429,44 & 4625,79 & 4633,65 & 0,103 \\
\hline MR-B & 4575,84 & 4585,66 & 4809,46 & 5025,07 & 5033,66 & 0,112 \\
\hline MR-G & 4952,15 & 4963,22 & 5208,79 & 5445,00 & 5454,62 & 0,126 \\
\hline MR-V & 5369,03 & 5413,11 & 5664,96 & 5923,90 & 5659,56 & 0,135 \\
\hline MR-VR & 5850,19 & 5894,23 & 6165,79 & 6448,52 & 6468,52 & 0,148 \\
\hline MR-R & 6228,15 & 6243,10 & 6560,33 & 6865,26 & 6878,27 & 0,163 \\
\hline MR-RI & 6748,88 & 6764,58 & 7109,81 & 7440,85 & 7454,46 & 0,172 \\
\hline MR-I & 7369,39 & 7386,53 & 7765,14 & 8127,95 & 8142,75 & 0,189 \\
\hline MR-Z & 8787,88 & 8810,52 & 9274,84 & 9698,97 & 9740,20 & 0,222 \\
\hline HR-R & 6397,62 & 6405,61 & 6602,59 & 6797,14 & 6804,87 & 0,098 \\
\hline HR-I & 8358,64 & 8380,20 & 8626,01 & 8882,38 & 8984,87 & 0,130 \\
\hline
\end{tabular}


Finally, as a summary of the daytime activities we show in Figure 8 the distribution of the images taken during those periods split by instrument mode and VPH setup. In total we obtained 851 images, 497 with the LCB and 345 with the MOS (pseudo-slit vignetting test images were neither LCB nor MOS images). By VPH, the most widely used units were LR-I (248 images), MR-VR (67), LR-R (56), LR-Z (49), MR-V (43) with about 20-30 images being taken for each of the other units. Besides, we completed $\sim 1000$ spectrograph movements and $\sim 50,000$ individual robotic positioners movemer-
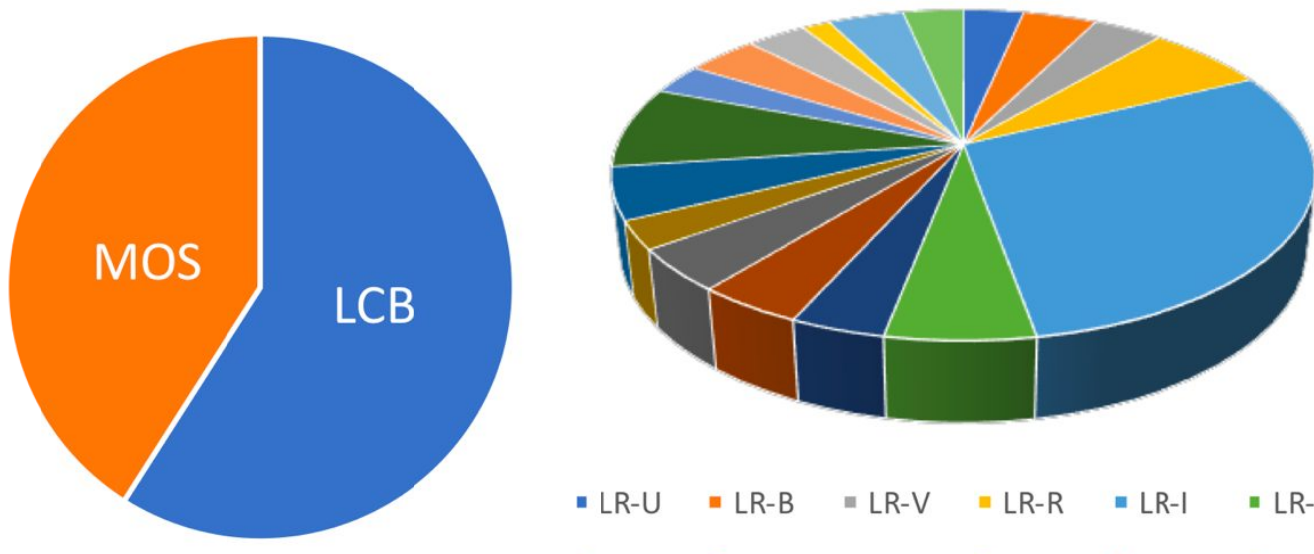

- LR-U "

- MR-U = MR-UB = MR-B = MR-G " MR-V " MR-VR

" MR-R = MR-RI $=$ MR-I $=M R-Z$ = HR-R $=H R-I$

Figure 8: Distribution of images by instrument mode and VPH setup taken during the daytime commissioning of MEGARA. The most commonly used VPH was the LR-I once since this was used extensively as part of the MEGARA Spectrograph setup tests described in the text.

\subsection{Night-time commissioning}

The night-time commissioning was carried in a total of 30 nights split in three 10 -nights observing runs, from June $24^{\text {th }}-$ July $3^{\text {rd }}$, July $24^{\text {th }}-$ August $2^{\text {nd }}$, and August $22^{\text {nd }}-$ August $31^{\text {st }} 2017$. Despite having nights with very high dust content in the atmosphere (calima), the weather was very good in terms of seeing and cloud coverage during the first two runs. The second half of the third run the weather was significantly worse, so roughly three nights could be considered as lost due to bad weather. Since A\&G unit of the FC-C had been commissioned by GTC right before the first nigh-time commissioning run, its behavior was a bit erratic which caused multiple shutdowns. The M3 mirror of GTC was also delivering a 5-arcmin misaligned pupil and it is not clear whether the problems in the M3 support pads found in November 2017 were responsible of this. Despite of that the commissioning observations were successful and the specifications of the instrument were verified on sky.

The first on-sky observation was taken on the night of Jun $24^{\text {th }} 2017$ by observing the spectrophotometric standard star and pre-planetary nebula ${ }^{10} \mathrm{BD}+33^{\circ} 2642$ with the LCB in the LR-R setup. and the official dedication of MEGARA was held at GTC on the night of July $25^{\text {th }} 2017$.

In this section we describe the results of the 12 night-time commissioning tests, while the scientific results derived from the astronomical targets observed as part of these tests are discussed in Section 3.3. The 12 night-time commissioning tests are the following:

- MEGARA telescope focus on lens array surface

- Optical-axis alignment

- MEGARA Folded-Cassegrain dynamical Tilt

- Telescope $+\mathrm{A} \& \mathrm{G}$ pointing corrections

- Dynamical Fiber MOS 
- $\quad$ LCB and Fiber MOS pointing

- Spaxel area and distance between spaxels (aka Spaxel size)

- $\quad$ Field of View

- Spectral performance

- Throughput and flux uniformity

- LCB Fast-mapping

- Spectroscopic limit

Below, we provide a brief description of the results of those tests that are either based on twilight observations or are tests where the results of the tests do not require of a direct scientific interpretation neither are of scientific use by themselves. Those tests (Spectral performance, LCB Fast-mapping) whose results rely on a more detailed scientific analysis of the data are described as part of the Early science results presented in Section 3.3.

\section{MEGARA telescope focus on lens array surface:}

The objective of this test was to determine the optimal focus of the telescope on the LCB lens array. By design, this would be also the optimal focus for the MOS observations. This was done by means of moving the GTC M2 mirror in steps in its $\mathrm{z}$ axis ${ }^{\mathrm{a}}$ while taking images of a bright star in between every focus configuration of the mirror. In Figure 9 we show the FWHM of the PSF the bright star observed as part of this test computed from a square-pixel image generated from the reduced RSS images of the star. As shown in this figure, the optimal telescope focus was only $\sim 0.01 \mathrm{~mm}$ the nominal focus at that temperature and telescope position.

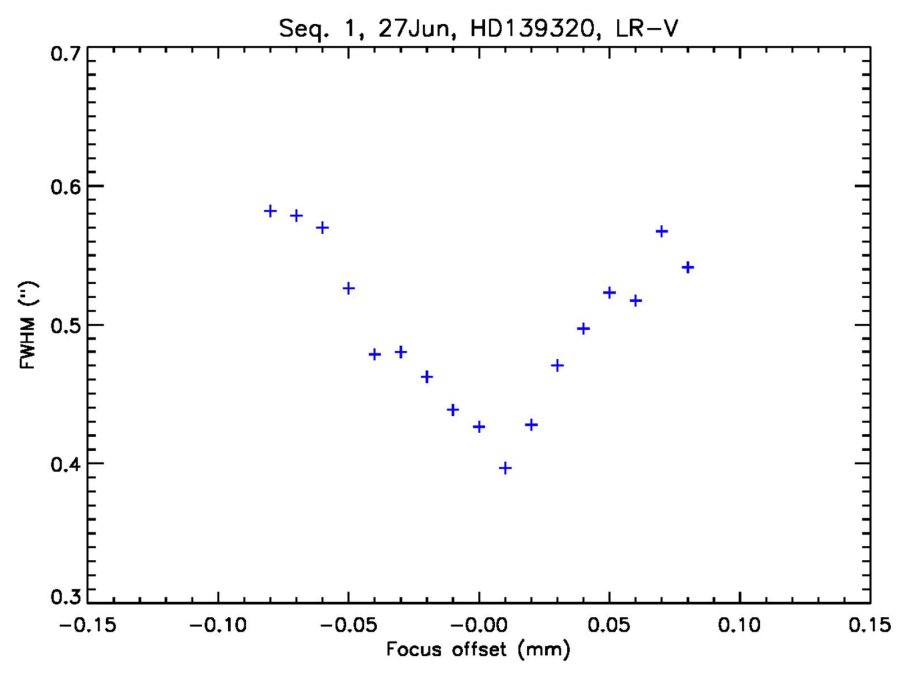

Figure 9: Variation of the FWHM with the focus offset. These measurements were taken on star HD139320 using the LCB with the LR-V VPH on the exceptional night in terms of seeing of June $27^{\text {th }} 2017$. Note that we were able to measure FWHM values as low as $\sim 0.4$ arcsec in the synthetic image of this star. For these seeing values the resulting FWHM must be taken with caution as the PSF is poorly sampled since the MEGARA LCB makes use of 0.62 -arcsec spaxels.

\footnotetext{
${ }^{a}$ The secondary mirror M2 is a lightweight convex hyperbolid of beryllium with aluminium coating. M2 defines the telescope pupil, and in order to minimize effects of thermal radiation its outer perimeter and central hole reflect the shape of M1. Its area is equivalent to a circular mirror with a diameter of $1.06 \mathrm{~m}$. For alignment with M1, M2 has 5 degrees of freedom: movement in $\mathrm{x}$ and $\mathrm{y}$ (decentering) and $\mathrm{z}$, i.e. focus (along the optical axis), as well as tip- tilt rotations. For chopping, the maximum frequency is 14 movements per second.
} 


\section{Optical axis alignment:}

The goal of this test is to assess the alignment of the optical axis performed during MEGARA integration.

To perform this assessment the fibers flux at different rotators angles were measured selecting the LCB mode. Measurements are taken at different rotator angles using dome flats and twilight images. The objective of the analysis is to identify if a tilt exists between the LCB and the GTC optical axis. A tilt between the instrument focal plane and the GTC optical axis would produce that not the same portion of the pupil would be entering at the fibers and, therefore, the total transmission would be different for the different angles of the FC-F rotator. Therefore, we took a series of 13 images with different rotator angles, separated in intervals of $30^{\circ}$, and evaluated the flux homogeneity within each of the 81 sets of 7 fibers constituting the LCB.

As reported in Pérez-Calpena et al. (2018a) ${ }^{9}$, the alignment of the MEGARA FC assembly with the FC-F rotator axis was verified to be below 0.5 arcmin with the boresight telescope. Otherwise, a misalignment of the FC-F optical axis of 5 arcmin was also measured. This sets a lower limit in the level of homogeneity in between our flux measurements.

Thus, in left panel of Figure 10 we show the maximum variation of flux in each minibundle of 7 fibers in the LCB. This maximum variation of flux per minibundle is computed as the percentage between the minimum and maximum flux per minibundle. The result is that the maximum variation is never larger than $5 \%$ with the minimum being $2 \%$ possibly due to the misalignment between the FC-F set optical and rotation axes indicated above.

\section{MEGARA Folded-Cassegrain dynamical tilt:}

The goal of this test is to identify any misalignment (if present) of the axis of any positioner out of its nominal position. We note that the dynamical alignment of each positioner had been evaluated during laboratory tests with the foreseen transmission homogeneity between different MOS mini-bundles (see Pérez-Calpena et al. $2018 \mathrm{~b}^{11}$ ). The procedure was first take an image with all MOS robotic positioners in their nominal positions. Then, we commanded one third the positioners to extend the robotic arm (R2 fully extended), so each of these positioners will be pointing to one point of the outer perimeter of the patrol area. Then, take an exposure. We moved again these positioners in the opposite direction ( $\mathrm{R} 2$ fully extended, $\mathrm{R} 1=180^{\circ}$ ), so each of them will be pointing to the opposite point of the patrol area perimeter. Then, take an exposure. We repeated the process with the other two thirds of the positioners. Finally, we checked the difference in flux between the images obtained with the R2 fully extended and that obtained in their nominal position. The results are shown in right panel of Figure 10. These values now represent the maximum flux variation for each MOS minibundle instead.

Thus, we verified that the results are in agreement with the dynamical tilt measurements performed at LICA-UCM per each positioner minibundle, with the maximum variation of flux per MOS minibundle being $\sim 10 \%$.
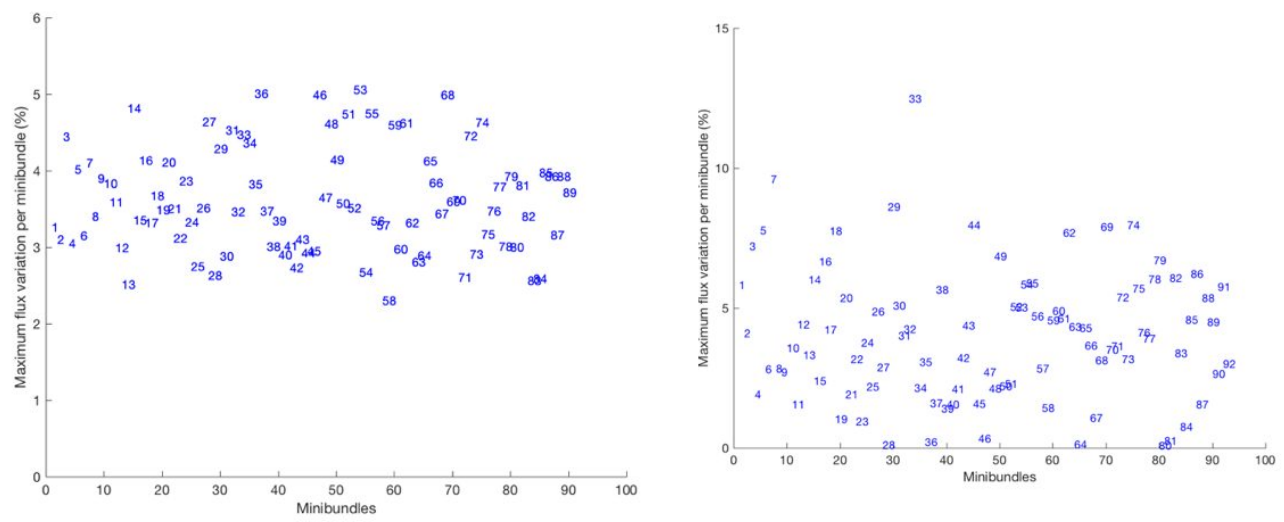

Figure 10: Left: Results of the optical axis alignment regarding the maximum flux variation per minibundle. Right: Results of the dynamical test regarding the maximum flux variation per minibundle. The labels indicate the IDs of the MOS minibundles. 


\section{Dynamical MOS test:}

Once that both the overall optical axis alignment for LCB and MOS set during integration ${ }^{9}$ and the dynamical tilt of the MOS measured at LICA-UCM are both verified using flux-variation arguments we had to test the precision of the $(\mathrm{x}, \mathrm{y})$ position nominal design values of every robotic positioner and its corresponding precision in placing its minibundle in the design position within the patrol area. In order to do that we observed a couple of Galactic Globular Clusters (M15 and M71) with enough stars brighter than Gaia $G=16$ mag to occupy the vast majority of the positioners in one single pointing. In left panel of Figure 11 we show the layout of the stars in M15 observed as part of this test as given by the FMAT tool included in the MOPSS. For both these clusters we obtained LR-R spectra of all stars selected. Raw images were reduced with the pipeline and analyzed to compute the centroid errors in (x,y) coordinates for each minibundle with respect to the center of the minibundle. These $(\mathrm{x}, \mathrm{y})$ errors obtained after the refinements in the Instrument Position Angle (IPA) given by GTC and in the acquisition procedure are also shown in Figure 11 (center). From the modules of the corrected error vectors given the right panel of Figure 11 we conclude that the average positioners pointing error is currently 0.17 arcsec. This could be improved to be within $0.1 \mathrm{arcsec}$ if the offsetting residual errors are corrected (i.e., current telescope offsetting accuracy is improved). The only positioner that shows errors $<0.2 \operatorname{arcsec}$ is $\# 34$. The average offsetting residual error after verifying pointing with the LCB is -0.06 ( $\mathrm{x}$ axis) and -0.11 (y axis).
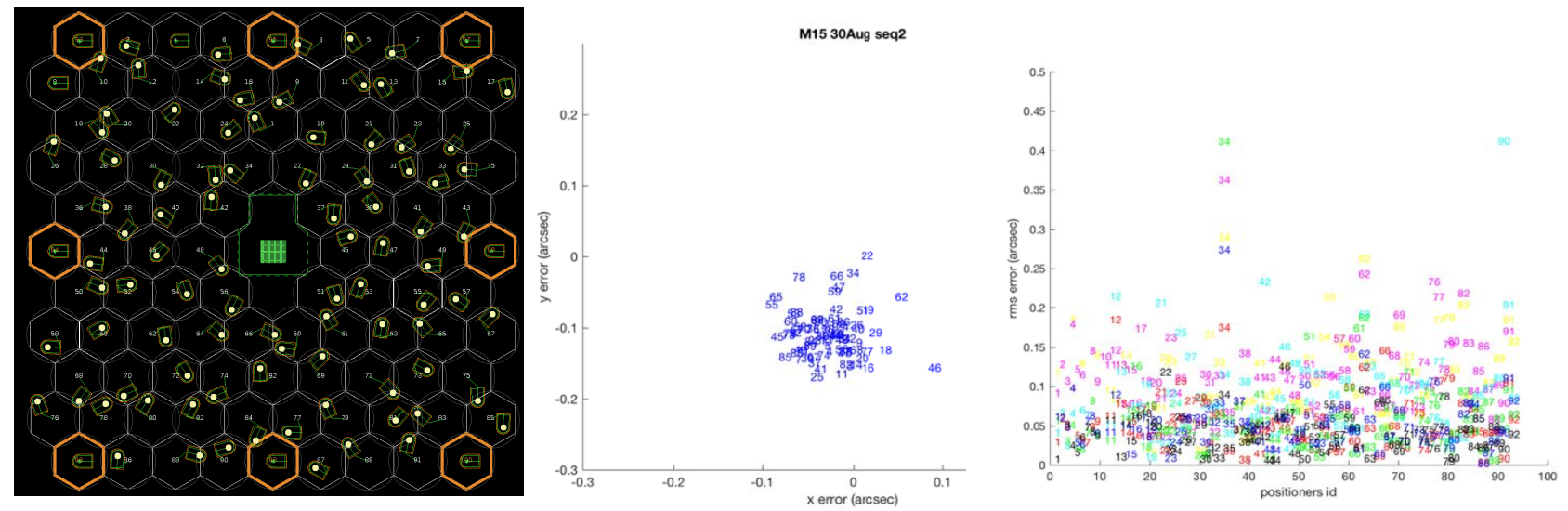

Figure 11: Left: Layout of the stars in M15 assigned to each of the positioners of the MOS. Center: Offsets derived from the stars observed in M15 on August $30^{\text {th }}$ 2017. Right: Modules of the error vector for all observed M15 and M71 configurations.

\subsection{Early-science}

Most of the night-time commissioning observations required of targeting specific astronomical targets. In Figure 12 below we show the distribution of targets by type that were observed as part of our three observing runs. The term "clusters" refers here to Galactic Globular Clusters, in particular M15 and M71, which, as mentioned above, were used to evaluate the pointing accuracy of the MOS robotic positioners. The rest of the targets observed were individual stars, nebulae (Galactic PNe or Local Group HII regions) or external galaxies.

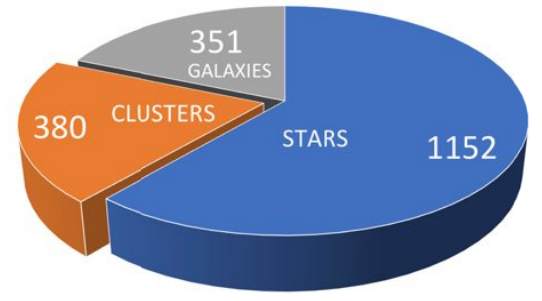

Figure 12: Distribution by type of those astronomical targets observed with MEGARA as part of its night-time commissioning. 
In the case of the stars, we observed (1) very bright single stars (for test Telescope + A\& $G$ pointing corrections), (2) multiple systems (for tests Spaxel area and distance between spaxels and Field of View), (3) spectrophotometric standards (for test Throughput and flux uniformity), or (4) stars of different spectral type selected from stellar libraries ${ }^{12}$ (for test Spectral performance). In order to evaluate the performance of the MEGARA LCB when observing extended nebular objects showing a wide range in surface-brightness and brightness contrast, as part of the Spectral performance test, we observed (1) three Planetary Nebulae (PNe), including (from faint to bright line emission) BD $+33^{\circ} 2642^{10}$, M192 and NGC7027. Among this same test we also explored (2) nearby galaxies covering a range in surface-brightness, morphology (from ellipticals to spirals also including quiescent and actively star-forming compact dwarfs) and distance (i.e. spatial resolution in physical units). Finally, we also verified the ability of carrying out mosaics combining multiple LCB IFU pointings by doing (3) a 2x2 pointings map of the massive HII region NGC604 in the Local Group galaxy M33 (test $L C B$ Fast-mapping).

In the rest of this section we will present some of the early results from the exploitation of the commissioning data on these targets.

\section{First light on $B D+33^{\circ} 2642$ :}

On June $24^{\text {th }} 2017$, MEGARA saw its first light while observing the spectrophotometric standard star BD $+33^{\circ} 2642$. This is a $\sim 10^{\text {th }}$-magnitude B2 IV star that is also an HST primary standard. Although it has been known to have some line emission (mainly in $\mathrm{H \alpha}$ but also in other fainter forbidden lines), it has been only recently the existence of an extended emission nebula around was reported ${ }^{10}$. Our 10 -seconds-long first-light image of BD+33 2642 in the LR-R setup actually revealed a ring-like nebula around the central star with a diameter beyond 5 arcsec showing line emission both in $\mathrm{H \alpha}$ and in the $[\mathrm{NII}] \lambda \lambda 6548,6584 \AA \AA \AA$ doublet (see Figure 13), which identifies this star as a possible pre-planetary nebula.

Follow-up LR-U and high-resolution MR and HR observations on this target confirmed the spectroscopic type of the central star showing also strong absorption in high-order Balmer lines, with even lines between H17-H20 being clearly detected in our LR-U 120 seconds-long spectrum (see Figure 14). These short observations already demonstrated, as part of the Spectral performance night-time commissioning test, that MEGARA is perfectly suited for observing extended faint nebular objects with resolutions from $\mathrm{R}=6,000-20,000$ all the way from $365 \mathrm{~nm}$ to almost $1 \mu \mathrm{m}$.
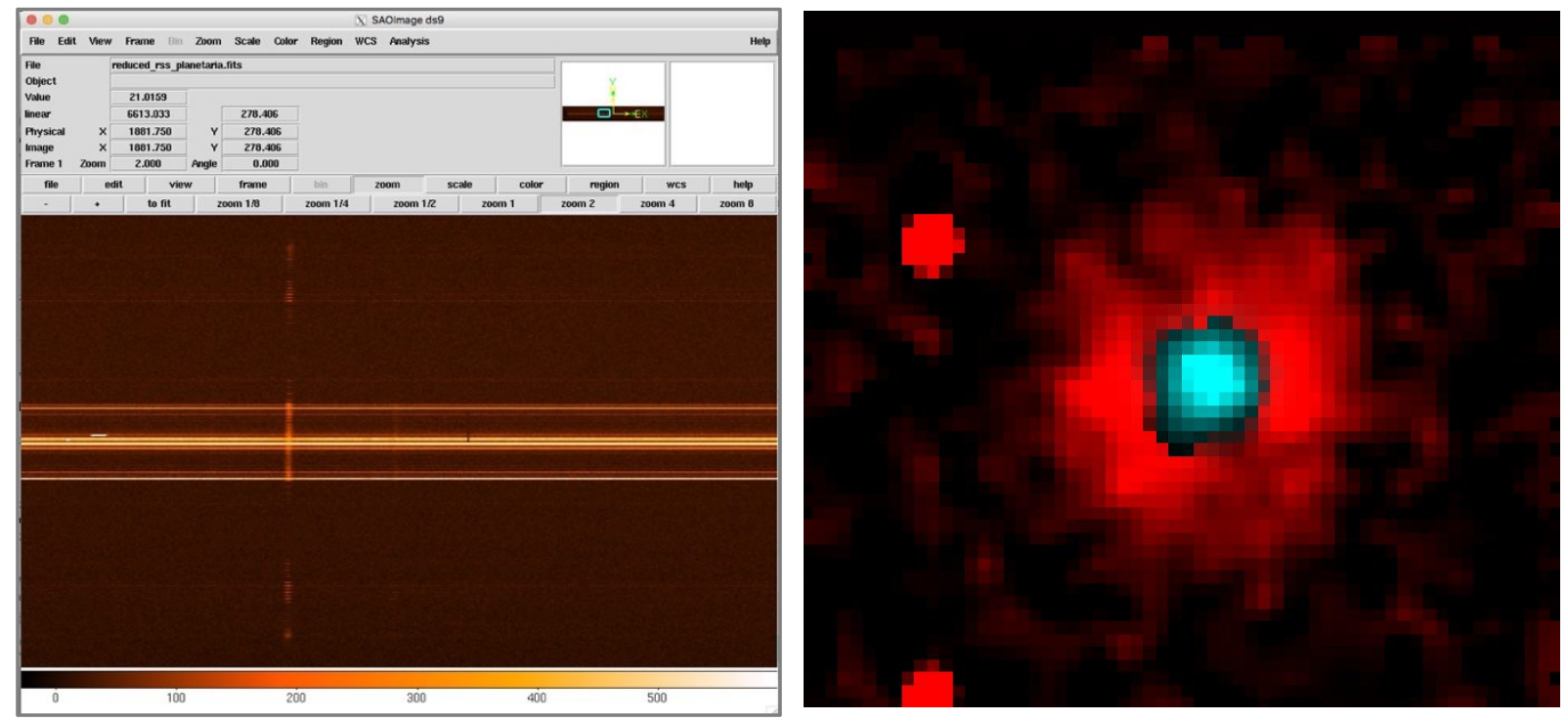

Figure 13: Left: First-light raw image of MEGARA on BD $+33^{\circ} 2642$ using the LR-R and an exposure time of only 10 seconds. Note that the line emission is detected in significantly more fibers than the continuum. Right: Synthetic RGB image of pre-planetary nebula $\mathrm{BD}+33^{\circ} 2642$. The distribution of the $\mathrm{H} \alpha$ line emission is shown in red (R channel) while the continuum in the R-band is shown in cyan (obtained as a combination of $\mathrm{G}$ and $\mathrm{B}$ channels). 


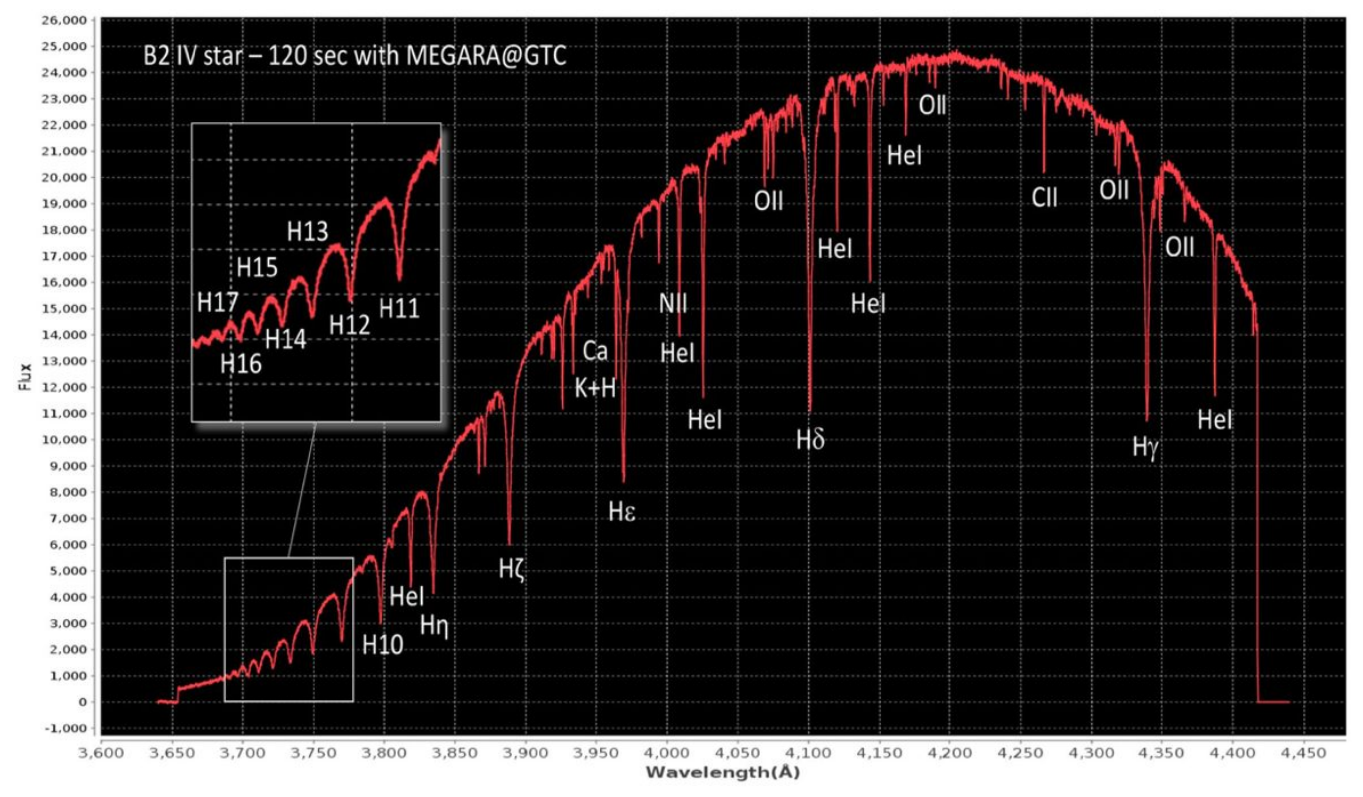

Figure 14: Blue spectrum of the central star of object BD $+33^{\circ} 2642$ obtained with the MEGARA LR-U VPH and a 120 seconds-long exposure. Note that this spectrum has not been corrected for the system sensitivity function.

\section{MEGARA Spectral library:}

As part of the commissioning observations of MEGARA we obtained spectra of stars of different spectral types and magnitudes. This allowed us (1) to evaluate the performance of the instrument for detecting different types of spectral features in different parts of the optical spectrum and (2) to start putting together the MEGARA Spectral Library (García Vargas et al. 2018, in prep.). In Figure 15 we show four spectra acquired as part of this library during commissioning.

\section{Reddened Galactic massive stars:}

In order to evaluate the performance of MEGARA for observing very red objects, we targeted J20395358+4222506, a strongly reddened star in the outer field of the Cyg OB2 association. The visual extinction is estimated to be $A_{V}=11$ mag. The apparent $\mathrm{B}$ magnitude from the USNO-B catalogue is $\mathrm{B}=15.9 \mathrm{mag}$, whereas the $\mathrm{K}_{\mathrm{s}}$ magnitude from 2MASS is as bright as $\mathrm{K}_{\mathrm{s}}=5.8 \mathrm{mag}$. This star has been recently classified by Berlanas et al. (2018) ${ }^{13}$ as a B0 I.

These properties resemble those of the extreme B4 Ia+ supergiant Cyg OB2-12, one of the most luminous and intriguing stars in the Galaxy and HD190603, a B1.5 Ia+ hypergiant. Both stars are very luminous, approaching or exceeding $\log \left(\mathrm{L} / \mathrm{L}_{\text {Sun }}\right)=6.0$ (see Clark et al., 2012 ${ }^{14}$ ). The left panel of Figure 16 gives a comparison of the Ha profiles of J20395358+4222506 (black), Cyg OB2-12 (HET observations, kindly provided by A. Pellerin, in red) and HD190603 (from the IACOB database, Simon-Diaz et al., $2011^{15}$, in green). We see that the H $\alpha$ profile of J20395358+4222506 indicates an extreme mass-loss and luminosity. The MEGARA spectrum was obtained with the LCB+HR-R grating and $3 \times 300$ seconds exposure time. The right panel of Figure 16 shows the spectrum between $4000 \AA$ and $4400 \AA$ obtained with the LCB+LR-U grating and $3 \times 900$ seconds, degraded to $\mathrm{R}=3,000$. The most important lines are marked. The Si IV lines, needed to determine the effective temperature through the Si ionization balance, are clearly seen.

\section{Kinematics of stars and the ISM in nearby galaxies:}

Another important objective of the commissioning tests was to evaluate the stability and homogeneity of the spectral PSF in order determine high-order momenta of the velocity distribution of stars in external galaxies. With this objective 
in mind we observed a series of nearby galaxies of different types, including ellipticals (M32), spirals (NGC7025, UGC10205) and compact dwarfs (J0159 + 0751, J1608 + 3528, SDSS J223036.79-000636.9, Mrk 324).
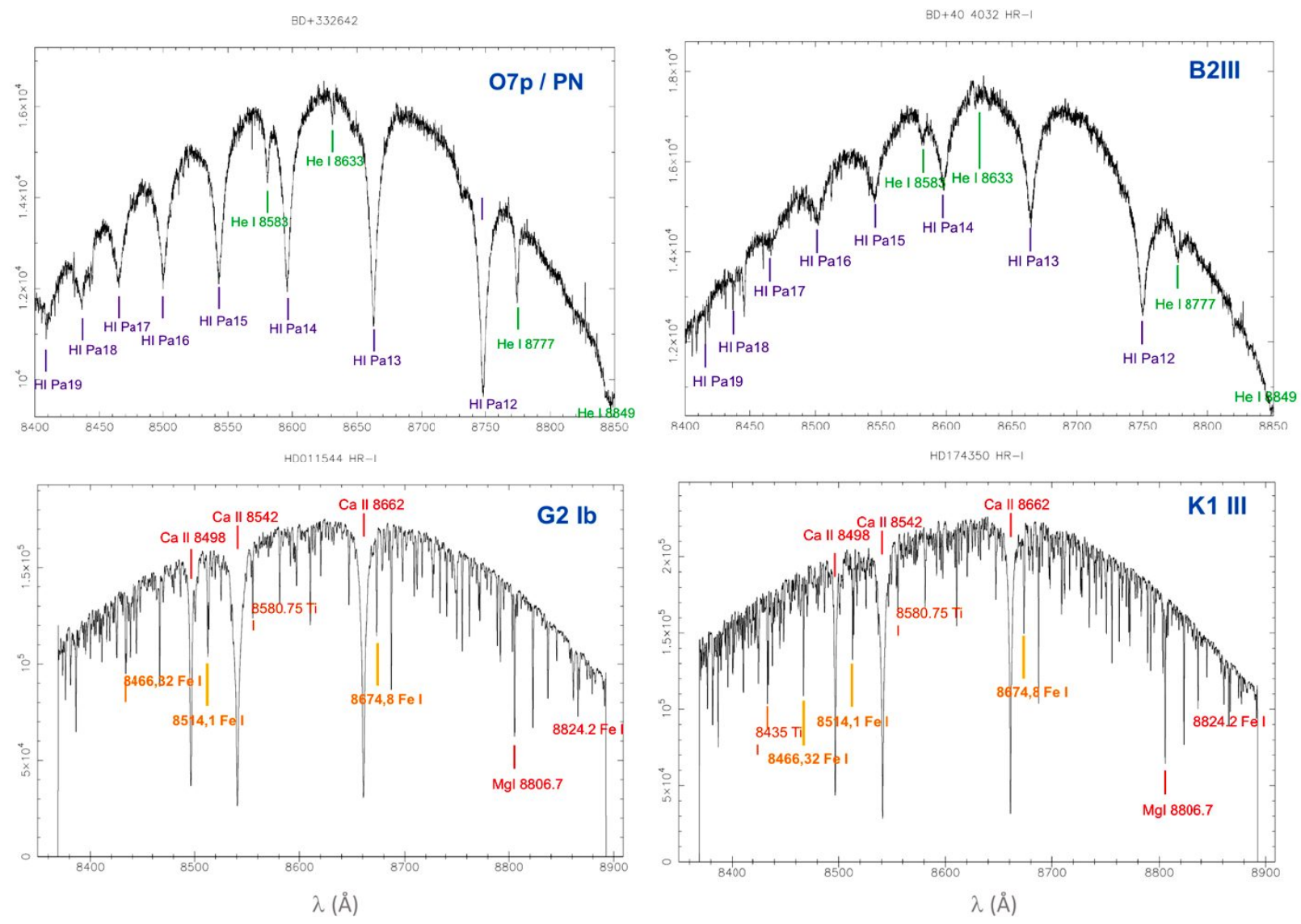

Figure 15: Spectra of four stars from the MEGARA Spectral library (García Vargas et al. 2018, in prep.) in the HR-I setup centered at the $\mathrm{CaT}$ with $\mathrm{R}=20,000$. Note that these spectra are not corrected for the instrument sensitivity function.
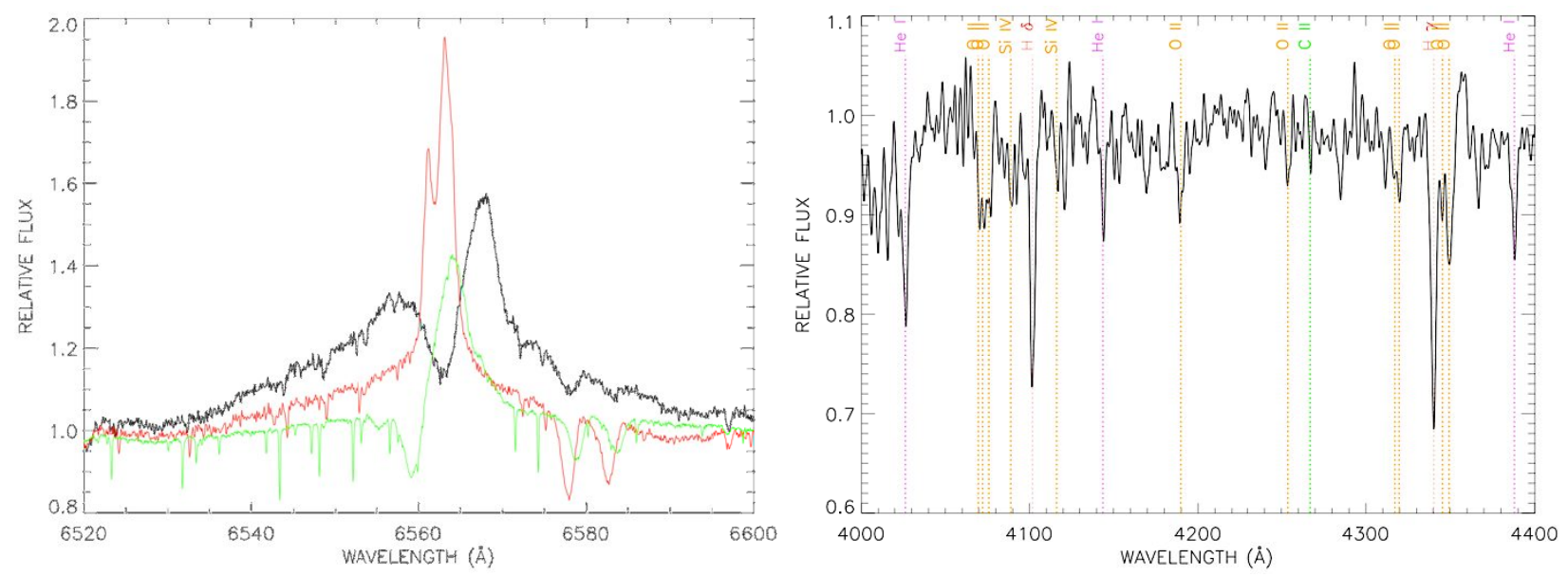

Figure 16: Left: Ha profiles of J20395358+4222506 (black; from LCB+HR-R observations), Cyg OB2-12 (red) and HD190603 (green). Right: LCB+LR-U spectrum (degraded to R=3,000) with an exposure time of 3x900 seconds. 

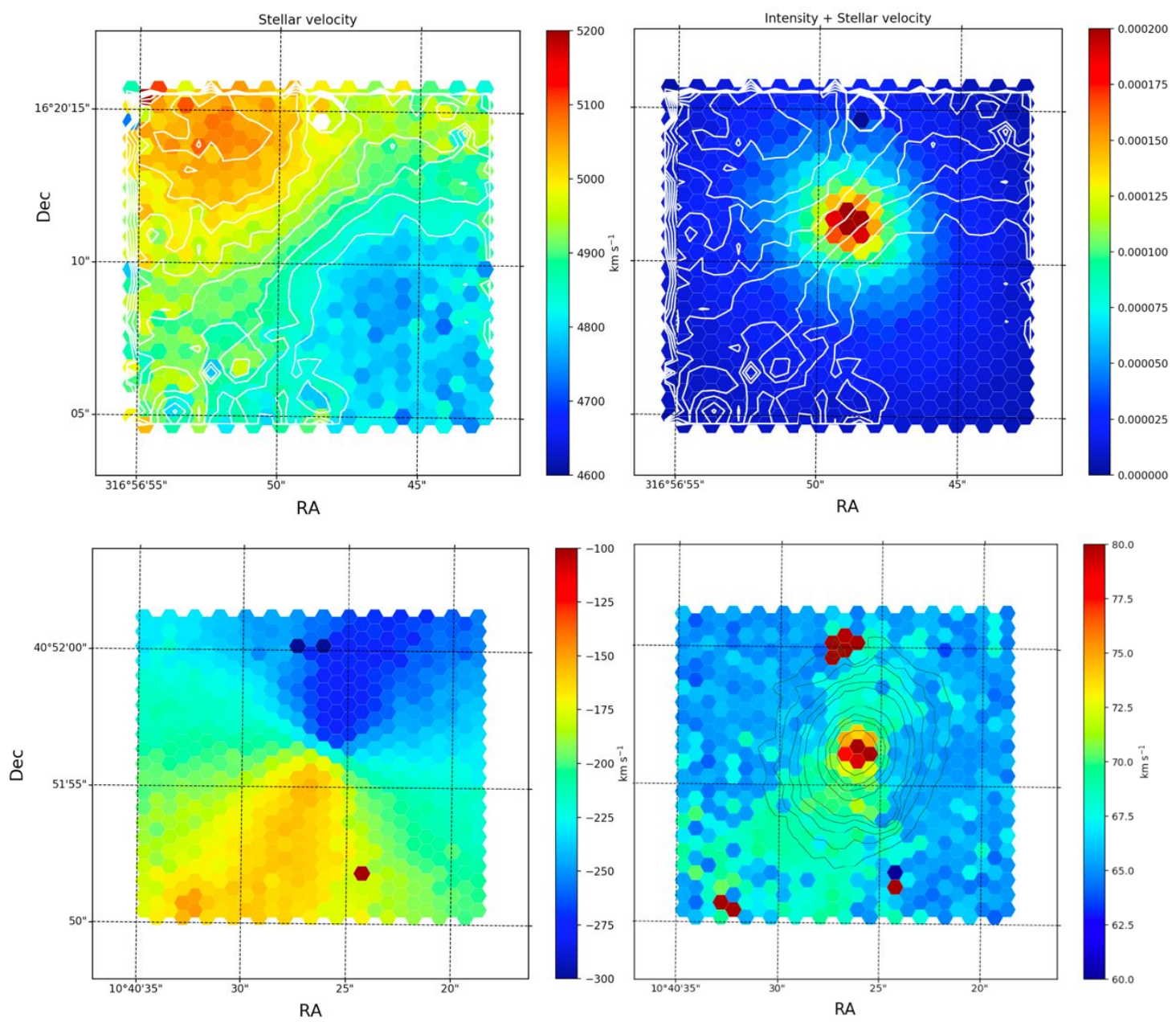

Figure 17: Kinematical analysis of NGC7025 (top) and M32 (bottom). The left panels show the stellar radial velocity, while the right panels show the V-band intensity map in the case of NGC7025 and the velocity dispersion map in the case of M32. In the case of NGC7025 the contours shown indicate iso-velocity curves obtained from its stellar velocity field while in M32 the contours correspond to the V-band continuum levels created from our LR-V observations.

In Figure 17 we show the results of the kinematic analysis of nearby galaxy NGC7025 and of the Local Group compact elliptical M32. The good image quality achieved, which is also fully characterized in 2D (i.e. as a function of wavelength and for all fibers), allowed us analyzing high-order Gauss-Hermite momenta, such as h3, h4 or even higher. In the case of NGC7025 the analysis of the stellar kinematics of its central regions based on one single LCB pointing in LR-V with $45 \mathrm{~min}$ exposure (and a physical resolution of $200 \mathrm{pc}$ ) shows evidence for fast rotation with associated cylindrical rotation and strong anti-correlation $\mathrm{V}-\mathrm{h} 3$ (see Falcón-Barroso et al. $2017^{16}$ ). Regarding the commissioning observations of M32 we find evidence for fast-rotation and, for the first time, we provide precise measurements of the kurtosis (h4) of the 2D LOSVD in M32.

\section{Distant galaxies and spectroscopic limit:}

In order to explore the limits of MEGARA for deep spectroscopy we considered different test cases, (1) the study of faint narrow emission lines, such as those arising from MW and Extragalactic Planetary Nebulae or Blue Compact Dwarf galaxies, (2) the study of intervening Lyman- $\alpha$ and metal absorbers in the spectra of background QSOs, (3) the 
observation of faint continuum emission and (4) the combined study of the stellar continuum of foreground red galaxies acting as gravitation lenses of foreground high-redshift galaxies.

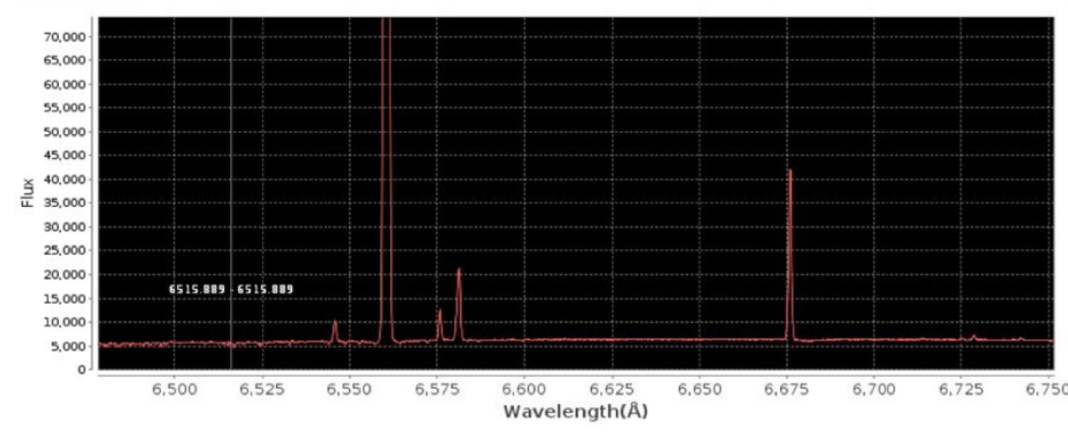

Figure 18: Top: LR-R spectrum of planetary nebula in the halo of $\mathrm{GC}$ M71 obtained with the MOS. Center: Spectrum and [OIII] 5007 image of BCD galaxy J223036.79-000636.9. Bottom left: Spectrum of $\mathrm{z}=3.62$ QSO B1422+2309. Bottom right: HST image of grav. lens J1630+4520 with continuum emission of $\mathrm{z}=0.248$ lens as yellow contours and [OIII] 5007 emission from the $\mathrm{z}=0.8$ lensed galaxy as white contours.
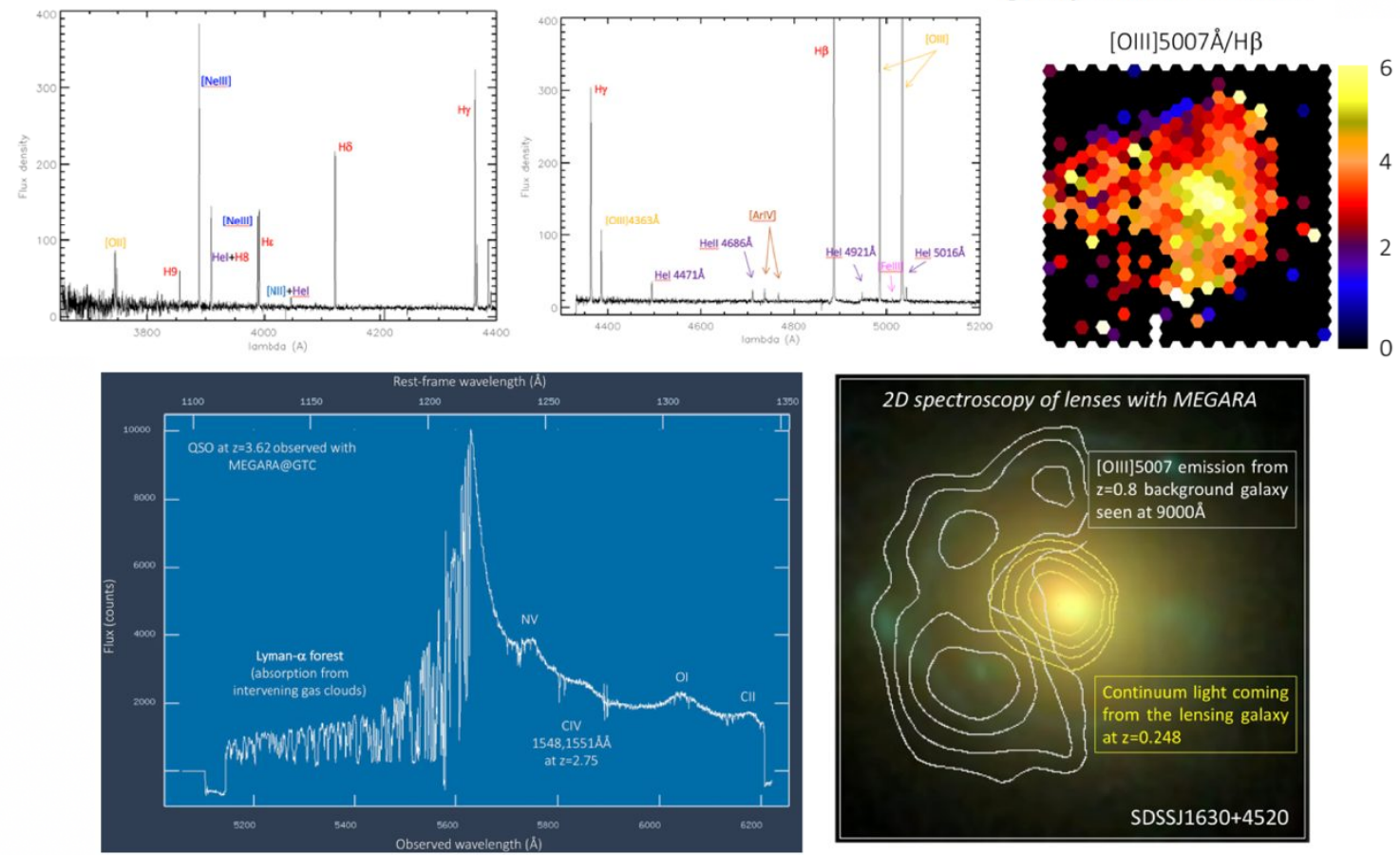

Figure 18 shows the results of some of these tests, including the emission (and continuum) spectrum of a PN in the halo of GC M71 observed with the MOS, the spectrum and [OIII] ] $\lambda 5007 \AA$ image of BCD galaxy SDSS J223036.79000636.9, LR-V spectrum of the Lyman- $\alpha$ Forest region of distant QSO ( $z=3.62) B 1422+2309$, and HST image of gravitational lens SDSS J1630+4520 with the continuum emission from the lens detected with MEGARA in the LR-Z setup shown as yellow contours and the amplified $[\mathrm{OIII}] \lambda 5007 \AA$ emission from background source at $\mathrm{z}=0.8$ shown as white contours.

\section{Distant galaxies and spectroscopic limit:}

Finally, in order to test the feasibility of acquiring mosaics of multiple pointings with the MEGARA IFU we obtained a 2x2 map of the central region of massive HII region NGC604 in the Local Group galaxy M33 in the LR-B VPH with an exposure time per pointing of only 5 minutes. A larger map covering the vast majority of the emission-line nebula has been proposed through Open Time. These observations allowed us to map already the gas kinematics and to identify the most massive stars in NGC604, some showing strong WR features and complex emission-line profiles (see Figure 19). 
Figure 19: MEGARA 2x2 mapping observations of NGC604@M33.

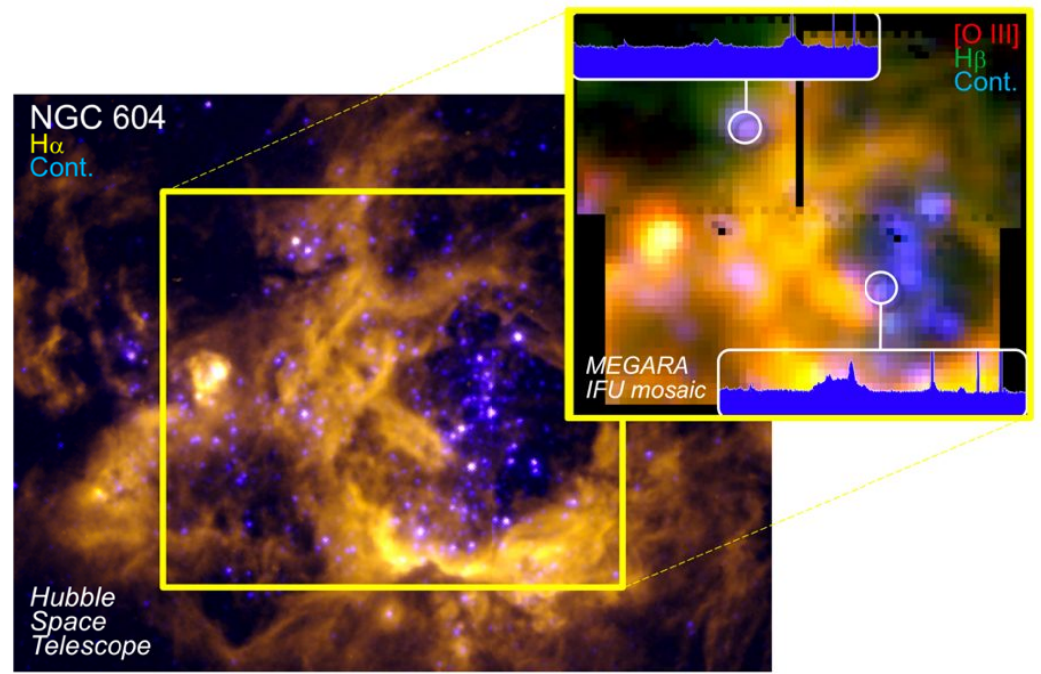

Our results and the accompanying contributions by Carrasco et al. $(2018)^{1}$, Pérez-Calpena et al. (2018a $)^{9}$, Pérez-Calpena et al. (2018b) ${ }^{11}$, GómezÁlvarez et al. $(2018)^{17}$, have showed that MEGARA fulfils all instrument and high-level scientific requirements and that it is a unique tool for carrying LCB IFU and MOS spectroscopy at $\mathrm{R}=6 \mathrm{k}-20 \mathrm{k}$ across the entire optical window (from $365 \mathrm{~nm}$ to $\sim 1 \mu \mathrm{m}$ ), which makes it particularly well suited for the study of narrow-lined targets.

\section{REFERENCES}

[1] Carrasco, E., et al., "MEGARA, the R=6000-20000 IFU and MOS of GTC", Proc. SPIE 10702-42 (2018)

[2] Gil de Paz, A., "MEGARA: the future optical IFU and multi-object spectrograph for the $10.4 \mathrm{~m}$ GTC telescope", Proc. SPIE Vol. 8446, 84464Q (2012)

[3] Gil de Paz, A., et al., "MEGARA: The future IFU \& MOS of the 10.4m GTC", Revista Mexicana de Astronomía y Astrofísica (Serie de Conferencias) 42, 90-92 (2013)

[4] Gil de Paz, A., et al., "MEGARA: a new generation optical spectrograph for GTC", Proc. SPIE, Vol. 9147, id. 914700 (2014)

[5] Gil de Paz, A., et al., "MEGARA, the new IFU and MOS for the GTC", Highlights of Spanish Astrophysics VIII, Proc. of the XI SEA Scientific Meeting. A. J. Cenarro et al. (eds.), p. 804 (2015)

[6] Gil de Paz, A., et al., "MEGARA, the new intermediate-resolution optical IFU and MOS for GTC: getting ready for the telescope", Proc. SPIE Vol. 9908, 99081K (2016)

[7] Sánchez-Contreras, C., Gil de Paz, A., Sahai, R., "The Companion to the Central Mira Star of the Protoplanetary Nebula OH 231.8+4.2", The Astrophysical Journal, Vol. 616, Issue 1., pp. 519-524 (2014)

[8] Sheth, K., et al., "The Spitzer Survey of Stellar Structure in Galaxies $\left(\mathrm{S}^{4} \mathrm{G}\right)$ ", Publications of the Astronomical Society of the Pacific, Vol. 122, Issue 898, pp. 1397 (2010).

[9] Pérez-Calpena, A., et al., "Delivery and integration of MEGARA at GTC: the process of going from laboratory to the telescope", Proc. SPIE 10705-13 (2018a)

[10] Napiwotzki, R., "BD+332642 - a new planetary nebula in the Galactic halo", Acta Astron., 43, 415 (1993)

[11]Pérez-Calpena, A., et al., "MEGARA MOS: where are my positions and fibres pointing to?", Proc. SPIE 10706$82(2018 b)$

[12] Sánchez-Blázquez, P., et al., "Medium-resolution Isaac Newton Telescope library of empirical spectral", Monthly Notices of the Royal Astronomical Society, Vol. 371, pp. 7013-718 (2006)

[13] Berlanas, S. R., et al., "New massive members of Cygnus OB2", A\&A, Vol. 612, id. A50, 20 pp. (2018)

[14] Clark, J. S., et al., "On the nature of candidate LBVs in M33", A\&A, Vol. 541, id. A146, 22 pp (2012).

[15] Simón-Díaz, S., et al., " IACOB spectroscopic database of Northern OB stars", SRSL Bulletin, Vol. 80, p. 514

[16] Falcón-Barroso, J., et al., "Stellar kinematics across the Hubble sequence in the CALIFA survey: general properties and aperture corrections", Astronomy \& Astrophysics, Vol. 597, id. A48 (2017)

[17] Gómez-Álvarez, P., et al, "MEGARA observation preparation \& QL software", Proc. SPIE 10707-56 (2018) 